\title{
Generalized Gas-Solid Adsorption Modeling: Single-Component Equilibria
}

Austin Ladshaw $^{\mathrm{a}}$, Sotira Yiacoumi*,a ${ }^{, \mathrm{a}}$, Costas Tsouris ${ }^{\mathrm{a}, \mathrm{b}}$, and David DePaoli ${ }^{\mathrm{b}}$

${ }^{a}$ School of Civil and Environmental Engineering, Georgia Institute of Technology, Atlanta, GA 30332-0355

${ }^{\mathrm{b}}$ Oak Ridge National Laboratory, Oak Ridge, TN, 37831-6181

*Corresponding Author: Telephone: 1-(404)-894-2639; Email: sotira.yiacoumi@ce.gatech.edu; Postal Address: Daniel Laboratory, 200 Bobby Dodd Way, Atlanta, GA, 30332-3055

Note: This manuscript has been authored by UT-Battelle, LLC under Contract No. DE-AC0500OR22725 with the U.S. Department of Energy. The United States Government retains and the publisher, by accepting the article for publication, acknowledges that the United States Government retains a non-exclusive, paid-up, irrevocable, world-wide license to publish or reproduce the published form of this manuscript, or allow others to do so, for United States Government purposes. The Department of Energy will provide public access to these results of federally sponsored research in accordance with the DOE Public Access Plan (http://energy.gov/downloads/doe-public-access-plan). 


\title{
Graphical abstract
}

\begin{abstract}
Over the last several decades, modeling of gas-solid adsorption at equilibrium has generally been accomplished through the use of isotherms such as the Freundlich, Langmuir, Tóth, and other similar models. While these models are relatively easy to adapt for describing experimental data, their simplicity limits their generality to be used with many different sets of data. This limitation forces engineers and scientists to test each different model in order to evaluate which one can best describe their data. Additionally, the parameters of these models all have a different physical interpretation, which may have an effect on how they can be further extended into kinetic, thermodynamic, and/or mass transfer models for engineering applications. Therefore, it is paramount to adopt not only a more general isotherm model, but also a concise methodology to reliably optimize for and obtain the parameters of that model. A model of particular interest is the Generalized Statistical Thermodynamic Adsorption (GSTA) isotherm. The GSTA isotherm has enormous flexibility, which could potentially be used to describe a variety of different adsorption systems, but utilizing this model can be fairly difficult due to that flexibility. To
\end{abstract}


circumvent this complication, a comprehensive methodology and computer code has been developed that can perform a full equilibrium analysis of adsorption data for any gas-solid system using the GSTA model. The code has been developed in $\mathrm{C} / \mathrm{C}++$ and utilizes a LevenbergMarquardt's algorithm to handle the non-linear optimization of the model parameters. Since the GSTA model has an adjustable number of parameters, the code iteratively goes through all number of plausible parameters for each data set and then returns the best solution based on a set of scrutiny criteria. Data sets at different temperatures are analyzed serially and then linear correlations with temperature are made for the parameters of the model. The end result is a full set of optimal GSTA parameters, both dimensional and non-dimensional, as well as the corresponding thermodynamic parameters necessary to predict the behavior of the system at temperatures for which data were not available. It will be shown that this code, utilizing the GSTA model, was able to describe a wide variety of gas-solid adsorption systems at equilibrium. Additionally, a physical interpretation of these results will be provided, as well as an alternate derivation of the GSTA model, which intends to reaffirm the physical meaning.

\author{
Abbreviations \\ GSTA Generalized Statistical Thermodynamic Adsorption \\ IAST Ideal Adsorbed Solution Theory
}

\title{
Keywords
}

Gas solid equilibrium; Computer modeling; Adsorption isotherm; Statistical thermodynamics 


\section{Nomenclature}

Latin Symbols

a parameter of the Freundlich isotherm relative to adsorption strength (Table 1)

A specific surface area of an adsorbent [Eq. (1)]

$b \quad$ parameter of the Tòth isotherm relative to heterogeneity and lateral interactions of molecules (Table 1)

$D \quad$ number of degrees of freedom in an equilibrium system [Eq. (12)]

$F \quad$ number of phases in an equilibrium system [Eq. (12)]

$f\left(x_{i}, \mathbf{t}\right) \quad$ generic representation of a model function [Eq. (11)]

$F_{o b j} \quad$ objective function used to penalize optimization with many parameters and/or few data points [Eq. (14)]

I number of intensive variables in an equilibrium system [Eq. (12)]

J Jacobian matrix of a model function

$K_{F} \quad$ parameter of the Freundlich isotherm relative to adsorption capacity (Table 1)

$K_{H} \quad$ Henry's Law constant at low pressure regime of an isotherm

$K_{L} \quad$ equilibrium constant of the Langmuir isotherm (Table 1)

$K_{L, n} \quad$ equilibrium constant at the $n^{\text {th }}$ adsorption site for the Heterogeneous Langmuir isotherm (Table 1)

$K_{L, n}{ }^{o} \quad$ dimensionless equilibrium constant at the $n^{\text {th }}$ adsorption site for the Heterogeneous Langmuir isotherm [Eq. (10)] 
$K_{n} \quad$ equilibrium constant of the GSTA model associated with the adsorption of $n$ adsorbate molecules [Eq. (2)]

$K_{n}{ }^{o} \quad$ dimensionless equilibrium constant of the GSTA model associated with the adsorption of $n$ adsorbate molecules [Eq. (3)]

$m \quad$ number of equilibrium parameters and adsorption sites, and/or adsorbed molecules, in the Heterogeneous Langmuir and GSTA models (Table 1)

M power representing the magnitude of the $K_{n}$ parameter of the GSTA model (Fig. 1)

$M \quad$ number of equilibrium data points of an experimental isotherm [Eq. (14)]

$n$ index for the number of parameters and adsorption sites, and/or adsorbed molecules, in the Heterogeneous Langmuir and GSTA models number of components in an equilibrium system [Eq. (12)] partial pressure of adsorbate species in the gas phase [Eq. (1)]

$P \quad$ total pressure of the system [Eq. (1)]

$P^{o} \quad$ standard state pressure, $100 \mathrm{kPa}$ [Eq. (3)]

$q \quad$ amount of adsorbate adsorbed at equilibrium [Eq. (1)]

$q_{\max } \quad$ maximum or monolayer capacity of the adsorbate-adsorbent system (Table 1)

$q_{n} \quad$ monolayer capacity of the $n^{\text {th }}$ adsorption site in the Heterogeneous Langmuir isotherm (Table 1) 
$q_{s} \quad$ monolayer capacity of each site in the Heterogeneous Langmuir isotherm if all sites have the same capacity

$R \quad$ gas law constant, 8.314 J/K mol [Eq. (1)]

$\mathrm{R}^{2} \quad$ correlation coefficient for a linear regression

$T \quad$ temperature of the system in Kelvin [Eq. (1)]

$\mathbf{t}$ parameter vector of a generic model function [Eq. (11)]

$x_{i} \quad$ independent variable of a model function at the $i^{\text {th }}$ data point [Eq. (11)]

$y_{i} \quad$ dependent variable of the $i^{\text {th }}$ data point [Eq. (11)]

\section{Greek Symbols}

$\Delta H_{L, n}{ }^{o} \quad$ standard molar enthalpy of adsorption at the $n^{\text {th }}$ adsorption site from the Heterogeneous Langmuir isotherm [Eq. (10)]

$\Delta H_{n}{ }^{o} \quad$ standard molar enthalpy of the adsorption of $n$ molecules [Eq. (4)]

$\Delta S_{n}{ }^{o} \quad$ standard molar entropy of the adsorption of $n$ molecules [Eq. (4)]

$\pi \quad$ spreading pressure of the system [Eq. (1)]

$\Phi \quad$ euclidean norm of the model function versus the data points [Eq.

\section{Code Symbols}

iso $\quad$ index for the isotherms given on input (Fig. A1)

m_dat number of data points for an experimental isotherm given on input (Fig. A1) 
max maximum number of parameters plausible for any isotherm of a system given the maximum number of parameters found applicable from the previous isotherm (Fig. A1)

$\mathrm{n} \_$iso number of experimental isotherms for a given adsorbateadsorbent system (Fig. A1)

n_par number of adjustable parameters being optimized for in the GSTA model (Fig. A1)

QMAX Boolean expression to check for the existence of the $q_{\max }$ parameter on input (Fig. A1)

qmax estimated value of the $q_{\max }$ parameter when not known or given as input (Fig. A1) 


\section{Introduction}

Gas-solid adsorption equilibria can be classified under a number of different categories depending on the details of the physical phenomena, such as physical adsorption versus chemical adsorption, or the topography of the adsorbent material (i.e., whether heterogeneous or homogeneous). Each of these various categories may require a different isotherm model to be used to describe the equilibrium between adsorbed $(q)$ and gas phases $(p)$ of a particular adsorption system [1]. From a scientific stand point, this is not only logical, but also a reasonable level of complexity as no single model can ever describe all possible systems. As a result, the most frequent approach for describing adsorption is through the utilization of one of the many common isotherm equations such as the Freundlich, Langmuir, and Tóth models $[1,2]$. Unfortunately, from an engineering point-of-view, the added complexity of having to seek out applicable models to a system makes it very difficult to standardize adsorption for design purposes and practical applications.

\subsection{Common Isotherm Models}

\subsubsection{Freundlich}

The Freundlich isotherm model, shown on Table 1, is typically regarded as the simplest model that can be used to represent adsorption equilibrium [1]. This is due in part to the ease in linearizing this equation in order to make correlations between the model and data to obtain the optimum parameters for that data set. Additionally, the parameters of the model have a simple 
interpretation: $K_{F}$ being relative of the capacity of adsorption and $a$ being relative to the strength of adsorption. However, this model is empirically derived [2] and would not represent any fundamental description of the adsorption phenomena.

\subsubsection{Langmuir}

While the Freundlich model is generally considered to be an empirical expression, the Langmuir model, also shown on Table 1, can be derived fundamentally by considering the following four assumptions: (a) there exist a fixed number of sites at which adsorption takes place, (b) all sites are identical, (c) each site only accommodates one adsorbate molecule, and (d) adsorbate molecules do not interact with each other. This derivation of the Langmuir model leads to the following physical interpretation of the parameters: $K_{L}$ is the equilibrium constant between the gas and adsorbed phases and $q_{\max }$ is the monolayer adsorption capacity of the adsorbent material. In addition, this equation can also be linearized, as shown on Table 1, in order to find the optimum parameter values for a given set of data. The simplicity of use combined with the clear physical significance of its parameters makes the Langmuir isotherm one of the most popular expressions of adsorption [1].

\subsubsection{Tóth}

Unlike the Freundlich and Langmuir models, which contained two adjustable parameters, the Tóth isotherm, shown on Table 1, is a three-parameter model that was extended from the Langmuir equation to consider the heterogeneity of adsorbent surfaces [2]. It is based on the 
experimental observation that heterogeneous adsorbents, of the same specific surface area, tend to uptake more adsorbate than an equivalent homogeneous adsorbent at the same pressure [3]. Under the circumstance in which the parameter $b$ is equal to one, the relative energies of the different adsorption sites are the same and the Tóth isotherm becomes the Langmuir isotherm.

While the exact physical interpretation of $b$ is unclear, it is believed to reflect the heterogeneity of the adsorbent, as well as the lateral interactions between adsorbed molecules [2,3]. The $K_{T}$ parameter is a function of the monolayer saturation pressure while $q_{\max }$ has the same meaning as in the Langmuir equation. Because of the similarities between the Langmuir and Tóth equations, the Tóth model is often utilized to correlate adsorption equilibrium data when the Langmuir model itself does not yield satisfactory results $[1,4]$.

\subsubsection{Heterogeneous Langmuir}

Similar to the Tóth isotherm, another heterogeneous adsorption model can be formulated as an extension of the Langmuir equation. If it can be assumed that the adsorbent surface acts as a patchwork of numerous, discrete homogeneous adsorption sites, then the Langmuir equation could apply separately to each individual site $[1,5]$. As a consequence, this means that each $n^{\text {th }}$ site is associated with its own equilibrium constant $\left(K_{L, n}\right)$ and adsorption capacity $\left(q_{n}\right)$. The resulting equation is referred to as the Heterogeneous Langmuir model, as shown on Table 1.

Although the Heterogeneous Langmuir model provides a simple and fundamental description of adsorption on heterogeneous surfaces, it is seldom used in practice due to the complexity involved in correlating this model with data and determining the number of discrete patches that make up the adsorbent material. As the number of patch sites $(m)$ increases, the number of 
necessary parameters to determine doubles and it becomes progressively more difficult to correlate the model with data. Furthermore, the Tóth isotherm model provides a simpler equation for describing the heterogeneity of surfaces by introducing only one additional parameter to the Langmuir model.

\subsection{Thermodynamic Considerations}

In addition to the dozens of isotherm models discussed in literature, there are also certain thermodynamic criteria, such as low- and high-pressure behavior, to be evaluated when considering the usage of a particular isotherm model. This behavior may dictate whether or not the model used in the correlation of the single-component data can be extended into theories for predicting multicomponent behavior $[1,2,6]$. Since any real adsorption application is likely to be with mixed gases, it is critical that single-component equilibria models meet the necessary set of criteria for extension into multicomponent models.

\subsubsection{Low Pressure Limits}

As the gas pressure in an adsorption system approaches zero, the relationship between the gas and solid phases should behave linearly. The low-pressure zone of adsorption is commonly referred to as the Henry Regime because the isotherm model becomes equivalent to Henry's Law (i.e., $q=K_{H} p$ ), where $K_{H}$ is the Henry's Law constant [1,6]. This behavior is particularly important with regards to the evaluation of the spreading pressure $(\pi)$ of the adsorbed phase, which must be evaluated between the limits of zero pressure and system pressure as follows: 


$$
\frac{\pi A}{R T}=\int_{0}^{P} \frac{q}{p} d p
$$

where $A$ is the specific surface area of the adsorbent, $R$ is the gas constant, $T$ is the temperature of the system, and $P$ is the total pressure of the system. If the adsorption isotherm does not have a finite limit at low pressure, then the spreading pressure of the system cannot be accurately determined, especially when considering low-pressure systems [6].

\subsubsection{High Pressure Limits}

Many monolayer adsorption theories, such as Langmuir, express the adsorbed phase quantity in terms of a theoretical maximum adsorption capacity [6]. In other words, using these theories, the amount adsorbed approaches the finite monolayer limit only if the pressures of the system become infinitely large (i.e., $\lim _{p \rightarrow \infty} q=q_{\max }$ ). This type of behavior is considered to be thermodynamically inconsistent and contrary to the Gibbs' thermodynamic expression of gassolid adsorption [2]. Realistically, the monolayer capacity should be obtainable at a finite saturation pressure as in the Tóth model. However, in practice it has been shown that the Langmuir model, and other monolayer theories, may still be applicable in describing equilibrium systems at pressures as high as 65 atmospheres ( 6.5 MPa) [7].

\subsection{Overview}

There are still dozens of more isotherm models available beyond what was discussed in Section 1.1, and each model has its own set of parameters that may all have different physical 
meanings and require a different correlation or regression method to provide the optimum values $[1,2]$. This makes adsorption modeling very impractical, as one would have to plan for nearly every possible scenario that could be encountered in natural and/or engineered systems. Furthermore, the extension of single-component models into multicomponent theories would be made extraordinarily difficult if different equilibrium models described each single-component isotherm, each with different parameters whose correlations with temperature are all different. Therefore, it is vital that adsorption modeling be generalized in a way that can accommodate the most variability reasonable within real and engineered systems.

In order to accomplish this objective, a generalized isotherm model must be adopted that can account for physical or chemical adsorption on either heterogeneous or homogeneous adsorbents. One particular model of interest is the Generalized Statistical Thermodynamic Adsorption (GSTA) isotherm model proposed by Llano-Restrepo and Mosquera [8]. In Section 2, the GSTA model derivation and significance will be briefly discussed. Furthermore, from its physical interpretation, we will provide an alternate derivation of the GSTA model based on the Heterogeneous Langmuir model. Since the GSTA model allows for a variable number of adjustable parameters, it could be utilized to correlate with a variety of different data sets. However, this flexibility makes it difficult to determine the number of parameters that would be most suitable in describing the data. In Section 3, the advantages and complications with using the GSTA model will be discussed, as well as the methodologies adopted to deal with these complications and make the GSTA model more readily usable by engineers and scientists. Lastly, Section 4 will show results from the GSTA model for an assortment of different gas-solid adsorption systems as a demonstration of the model versatility and generality. 


\section{Generalized Statistical Thermodynamic Adsorption Model}

The GSTA model presented by Llano-Restrepo and Mosquera [8] was originally presented as an alternate form of Hill's statistical model. Their aim was to provide a physical significance and reinterpretation of the parameters of Hill's model in such a way that they could be correlated with temperature to obtain site-specific enthalpies and entropies. The GSTA model and its parameters are summarized below:

$$
\begin{aligned}
& q=\frac{q_{\max }}{m} \frac{\sum_{n=1}^{m} n K_{n} p^{n}}{1+\sum_{n=1}^{m} K_{n} p^{n}} \\
& K_{n}=K_{n}^{o} /\left(P^{o}\right)^{n} \\
& \ln K_{n}^{o}=-\frac{\Delta H_{n}^{o}}{R T}+\frac{\Delta S_{n}^{o}}{R}
\end{aligned}
$$

where $m$ is the number of different types of adsorption sites available to adsorbate molecules, $K_{n}$ is the equilibrium constant relative to the adsorption of $n$ molecules in a network of available adsorption sites, $K_{n}{ }^{o}$ is the dimensionless equilibrium constant, $P^{o}$ is standard state pressure (100 $\mathrm{kPa}), \Delta H_{n}{ }^{o}$ is the standard enthalpy of adsorption of $n$ molecules in a network of sites, and $\Delta S_{n}{ }^{o}$ is the standard entropy of adsorption of $n$ molecules in a network of sites. The model contains $2+$ $m$ parameters $\left(q_{\max }, m\right.$, and $\left.K_{n}\right)$ that must be determined through correlations with equilibrium data, but if some information is known about the adsorbent, such as theoretical capacity and heterogeneity, then one could independently provide the $q_{\max }$ and $m$ parameters [8]. 


\subsection{Relation of the GSTA Model with the Heterogeneous Langmuir Model}

From the physical interpretation of the GSTA model provided by Llano-Restrepo and Mosquera [8], it can be inferred that this model is analogous to a heterogeneous site model in which each adsorption site is energetically distinct, but has the same capacity. To explore this idea further, consider a simple two-site Heterogeneous Langmuir model shown below in Eq. (5). If it is assumed that each adsorption site has the same capacity, as in the GSTA model, then it can be concluded that $q_{1}=q_{2}=q_{s}$ and therefore $q_{\max }=q_{1}+q_{2}$ or $q_{\max }=2 q_{s}$.

$$
q=\frac{q_{1} K_{L, 1} p}{1+K_{L, 1} p}+\frac{q_{2} K_{L, 2} p}{1+K_{L, 2} p}
$$

Expanding out Eq. (5), while utilizing the assumption of site capacity equivalence, results in the following:

$$
q=q_{s} \frac{K_{L, 1} p+K_{L, 2} p+2 K_{L, 1} K_{L, 2} p^{2}}{1+K_{L, 1} p+K_{L, 2} p+K_{L, 1} K_{L, 2} p^{2}}
$$

By further reducing and grouping like terms from Eq. (6), the model can be rewritten as:

$$
q=q_{s} \frac{\left(K_{L, 1}+K_{L, 2}\right) p+2\left(K_{L, 1} K_{L, 2}\right) p^{2}}{1+\left(K_{L, 1}+K_{L, 2}\right) p+\left(K_{L, 1} K_{L, 2}\right) p^{2}}
$$

From Eq. (7), the grouped terms can be renamed to formulate the equivalent terms from the GSTA model as the following: $K_{1}=\left(K_{L, 1}+K_{L, 2}\right)$ and $K_{2}=\left(K_{L, 1} K_{L, 2}\right)$. Lastly, by replacing the $q_{s}$ term with its $q_{\max }$ equivalent, Eq. (7) becomes Eq. (8) below, which is exactly equal to the GSTA model when $m=2$ (i.e., two-site adsorption).

$$
q=\frac{q_{\max }}{2} \frac{K_{1} p+2 K_{2} p^{2}}{1+K_{1} p+K_{2} p^{2}}
$$


This same derivation can be applied to any number of sites in the Heterogeneous Langmuir model and the results would be the same. Each $K_{n}$ term in the GSTA model results in a combinatorial factor of the $K_{L, n}$ parameters in the Heterogeneous Langmuir. For example, the $K_{n}$ parameters for the GSTA model for three adsorption sites would be as follows:

$$
\begin{aligned}
K_{\mathbf{1}} & =\sum_{n} K_{L, n} \\
K_{\mathbf{2}} & =\frac{\mathbf{1}}{\mathbf{2}} \sum_{n \neq k} K_{L, n} K_{L, k} \\
K_{\mathbf{3}} & =\prod_{n} K_{L, n}
\end{aligned}
$$

Therefore, the GSTA model is basically a special case of the Heterogeneous Langmuir isotherm in which each adsorption site has the same capacity for adsorption, but different energy characteristics.

\subsection{Physical Significance}

Considering that the GSTA model can be derived from the Heterogeneous Langmuir isotherm, constructing a physical interpretation of its parameters would be relatively straightforward. It can be concluded from Section 2.1 that both the $q_{\max }$ and $m$ parameters from the GSTA model were correctly defined by Llano-Restrepo and Mosquera [8] as the maximum adsorbent capacity and number of different types of adsorption sites, respectively. However, creating the link between the $K_{n}$ parameters and the standard enthalpy and entropy, as in Eq. (4), will require a deeper investigation.

For the Heterogeneous Langmuir model, the $K_{L, n}$ parameters are defined as the equilibrium constants associated with the adsorption of molecules at the $n^{\text {th }}$ site. As such, each site would 
have a different energy associated with it $[1,9]$ and could be correlated with temperature according to the van't Hoff equation [Eq. (10)], in which $K_{L, n}{ }^{o}$ is the dimensionless equilibrium constant of the Heterogeneous Langmuir model and $\Delta H_{L, n}{ }^{o}$ is the standard enthalpy associated with the $n^{\text {th }}$ site.

$$
\left(\frac{\partial \ln K_{L, n}^{o}}{\partial T}\right)_{n}=\frac{\Delta H_{L, n}^{o}}{R T^{2}}
$$

Integration of Eq. (10) will result in an equation, which takes the same form as Eq. (4), relating the $K_{n}$ parameters with temperature. Since the $K_{n}$ parameters are a function of the $K_{L, n}$ parameters as in Eq. (9), a direct leap between Eq. (10) and Eq. (4) cannot be made for the GSTA parameters. However, the relationship can be inferred through consideration of the particular combinations of $K_{L, n}$ that result in the $K_{n}$ parameters.

Recall from Section 2.1 the two-site Heterogeneous Langmuir model that resulted in the equivalent GSTA model. Each $K_{L, n}$ parameter is associated with a specific adsorbate-site interaction that can accommodate the same number of molecules. Therefore, for a pure gas with a two-site adsorbent, there are two distinct ways in which the interaction of a single molecule may occur: one molecule interacts with site one $\left(K_{L, 1}\right)$ or one molecule interacts with site two $\left(K_{L, 2}\right)$. Based on combinatorial mathematics [10], this particular event may be represented by the Rule-of-Sum, which states that any event whose tasks cannot be performed simultaneously can be accomplished as a sum of those tasks. In other words, the event which corresponds to the adsorption of a single molecule from the two-site model can be represented as the sum of $K_{L, l}$ and $K_{L, 2}$ (i.e., Event $1=K_{L, 1}+K_{L, 2}$ ).

The second event to consider is when two molecules of adsorbate are adsorbed, one to each available site (i.e., one molecule adsorbed to site one and site two or one molecule adsorbed to 
site two and site one). By combining the Rule-of-Sum with the Rule-of-Product [10], the second event can be represented as the sum of the product of the individual tasks. This results in the following: $K_{L, 1} K_{L, 2}+K_{L, 2} K_{L, 1}$. However, since the two products are indistinguishable from each other (i.e., $K_{L, 1} K_{L, 2}=K_{L, 2} K_{L, 1}$ ), they would be considered the same task and the duplicate must be eliminated. Therefore, the event is reduced to the following: Event $2=K_{L, 1} K_{L, 2}$.

From these definitions, it can be seen that events one and two correspond directly with the $K_{l}$ and $K_{2}$ parameters that were formulated in Section 2.1 from the Heterogeneous Langmuir model. This stands to reaffirm the physical interpretation of those parameters offered by Llano-Restrepo and Mosquera [8], who had claimed that the $K_{n}$ parameters represented the equilibrium constants associated with the adsorption of $n$ molecules into a collection of adsorption sites. Furthermore, because the parameters of the GSTA model are established as equilibrium constants, it is suitable to utilize the van't Hoff equation [Eq. (10)] in order to formulate their dependence with temperature as shown in Eq. (4). However, it is noteworthy to point out that the energy terms, which correspond to the equilibrium constants of the GSTA model, are not associated with site specific energies as in the Heterogeneous Langmuir model, but are instead representative of the event energies, which result from the adsorption of $n$ molecules in the site network of the adsorbent. 


\section{Utilization of the GSTA Model in Equilibrium Data Correlations}

\subsection{Advantages and Complications}

The greatest advantage of the GSTA model is in the fact that the equation contains an adjustable number of equilibrium parameters that may be used to describe the system. Logically, the more parameters a model is allowed to have, the better it can describe the system it is being applied to. However, this advantage leads to a number of complications with regards to the utilization of the model. Such complications include determining the maximum number of parameters allowed and the minimum number of parameters needed to describe the system.

Another advantage of the GSTA model is in the physical interpretation of the parameters themselves. After obtaining the appropriate equilibrium parameters from the system, a simple linear regression across temperature for the corresponding $K_{n}$ parameters will yield the relevant energy terms [Eq. (4)] necessary to predict the adsorption behavior between the temperatures for which experimental data are available. Therefore, as an additional complication, this requires that the model have the same number of parameters for each isotherm in a given adsorbateadsorbent system.

These complications and others were dealt with by using the techniques discussed in Section 3.2 below. The application of these techniques was accomplished in the development of a comprehensive $\mathrm{C} / \mathrm{C}++$ computer code (Appendix 1) to handle the variability of systems through a generalized treatment of each problem including whether or not $q_{\max }$ is known, the adsorbent's heterogeneity or homogeneity, and agnostic treatment of the units of adsorption capacity. 


\subsection{Solution Techniques}

\subsubsection{Non-Linear Least Squares Regression}

The form of the GSTA equation is indefinite and non-linear and therefore requires the use of a non-linear regression technique to accommodate the optimization of the parameters within the model. There are a number of non-linear optimization routines available (e.g., Newton's method, steepest-decent, trust region, ect.), but for the specific purpose of data correlation, a least squares method would be most applicable [11].

In any least squares method, the objective is to minimize the euclidean norm, or vertical displacement, between the y-axis data points and the result of the model function given the parameters and corresponding $\mathrm{x}$-axis points. The mathematical form of the euclidean norm is as follows:

$$
\Phi=\sqrt{\sum_{i}\left(y_{i}-f\left(x_{i}, \mathbf{t}\right)\right)^{2}}
$$

where $\Phi$ is the euclidean norm, $y_{i}$ is the $i^{\text {th }} \mathrm{y}$-axis data point, $f\left(x_{i}, \mathbf{t}\right)$ is the model, $x_{i}$ is the $i^{\text {th }} \mathrm{x}$ axis data point, and $\mathbf{t}$ is the parameter vector of the model.

\subsubsection{Determining the Number of Parameters}

The size of the solution vector for the GSTA equation represents the number of equilibrium parameters of the model. Note that the $q_{\max }$ parameter is not considered to be adjustable as it represents the theoretical maximum adsorption capacity for a particular adsorbate-adsorbent 
system. Without any knowledge as to the heterogeneity of the adsorbent, the logical course of action is to search for all parameter solutions for every applicable size of the solution vector and compare each solution to determine which describes the data best. Computationally this is very time consuming, but can be optimized by careful elimination of unnecessary solution searches and setting up an objective function whose purpose is to minimize the size of the solution vector.

An obvious approach to limiting the number of parameters in the model is to consider the number of data points that the model is attempting to describe. Mathematically, it is impossible to describe any set of data with a number of parameters that exceeds the number of data points $(M)$, but this can be taken a step further by considering that the number of parameters should be only a fraction of the number of data points. One could adopt a Two-Fifths compromise to place an absolute limit on the number of parameters to two-fifths of the total number of data points (e.g., $m \leq 2 / 5 M)$. However, this method is entirely arbitrary.

An alternate method to limit the total number of parameters could be adopted by using the Gibbs' Phase Rule [12,13]. Eq. (12) below can be used to determine the degrees of freedom $(D)$ for an equilibrium system from the number of components $(N)$, phases $(F)$, and intensive variables $(I)$. For gas-solid adsorption systems there are two components (adsorbate and adsorbent), three phases (gas, solid, and adsorbed), and three intensive variables (pressure, temperature, and spreading pressure), therefore the number of degrees of freedom would be two [12]. Additionally, the Gibbs' Phase Rule can also be applied in determining the number of equilibrium parameters $(m)$ applicable to describe the system. From Eq. (13), it can be concluded that the maximum number of allowable equilibrium parameters to describe a gas-solid adsorption system is six. However, this rule does not yield any information as to the minimum number of parameters that may be able to describe the system. 


$$
\begin{aligned}
& D=N-F+I \\
& m=(N-\mathbf{1}) F+I
\end{aligned}
$$

Minimizing the number of parameters in the GSTA model will simply reduce the calculations and the possibility of overfitting or over describing a data set with unnecessary parameters. This can be accomplished by creating an objective function that serves to penalize data correlations that have a large number of parameters or a small number of data points. Such an objective function would be as follows:

$$
F_{o b j}=\sqrt{\frac{\sum_{i}\left[\left(y_{i}-f\left(x_{i}, \mathbf{t}\right)\right) / y_{i}\right]^{2}}{M-m-1}}
$$

whose parameters are the same as in the euclidean norm of Eq. (11). While the overall adequacy of the model to represent the data would be determined by the euclidean norm, the most suitable solution with the least number of parameters would be determined by the objective function. Therefore, the objective function would serve as the final criterion in determining the number of parameters in the GSTA model.

A systematic procedure necessary to find the most suitable number of parameters is to start with the minimum number of parameters $(m=1)$, find an applicable solution via non-linear least squares regression, then increment the number of parameters by one. At each stage, the values of the euclidean norm and objective function are observed and compared to the previous stage. This process is continued until the maximum number of parameters applicable to the system has been reached (i.e., when $m=2 / 5 M$ or $m=6$, whichever is smaller). Finally, the most suitable solution is determined by locating the smallest $F_{o b j}$ from all solutions found. 


\subsubsection{Educated Initial Value Guessing}

Unlike linear least squares regression, a non-linear regression analysis must start with an initial guess being made to the solution vector $(\mathbf{t})$. Then, gradient observations must be made through the use of Jacobian matrices $(\mathbf{J}=\partial f / \partial \mathbf{t})$ in order to establish a magnitude and direction in which to shift the parameters of the model. This procedure is repeated until a reasonable solution is located, but due to the nature of non-linear regression, the ability to obtain solutions can be dependent on the initial values given in the solution vector [11]. To combat this issue, a number of techniques are adopted to ensure that the initial guess vector is as close to the solution as possible and that guesses being made are not arbitrary or irrelevant in magnitude.

The first guess is realized when considering that the GSTA model is exactly equal to the Langmuir equation when the number of equilibrium parameters $(m)$ is equal to one. Under this condition, it is possible to linearize the equation (see Table 1) and perform a standard linear regression to obtain the parameters. The optimized parameters from the linear regression are then used in the non-linear analysis as the initial guess for the parameter vector.

The previously optimized parameters from the one-parameter solution found are then used as a basis for making the initial guess for the two-parameter model. Using that information, the two unknown parameters are given an initial value equal in magnitude to the previously found one parameter. Maintaining the relative magnitudes in all parameters will ensure that no parameter is seen as irrelevant in the current iteration and will therefore be treated as equally important in the overall model.

All subsequent guesses, for three parameters and beyond, are made as order-of-magnitude slope projections from two previously optimized parameters and the new parameter in the vector 
is a slope projection from the other parameters within the vector. For example, if the current iteration is meant to find a three-parameter $(m=3)$ solution, then the initial guess for the $K_{l}$ parameter will be based on the optimized $K_{l}$ parameters from the two- and one-parameter solutions, the $K_{2}$ parameter will also be based on that slope, and the $K_{3}$ parameter will be based on the slope between the $K_{l}$ and $K_{2}$ parameters that for the current iteration. Fig. 1 provides a visualization of this procedure.

\subsubsection{Handling Multiple Isotherms}

All previously mentioned techniques (Section 3.2) have been applied to a single isotherm set of data, but most gas-solid adsorption equilibria data for a system are composed of many isotherms. Each isotherm within the same system must have the same value for $q_{\max }$ and be described by the same number of parameters in order to make any correlations with temperature (recall Section 3.1) to obtain the relevant energy terms $\left(\Delta H_{n}{ }^{o}\right.$ and $\left.\Delta S_{n}{ }^{\circ}\right)$. Accomplishing this feat requires an additional procedure that can work alongside all other techniques.

From Section 3.2.2, the number of parameters that best describes each isotherm can be determined independently, but there needs to be a way to determine the number of parameters that can describe all isotherms. Each isotherm begins searching for solutions to the simplest model $(m=1)$, then continues to add complexity $(m=m+1)$ until solutions are no longer considered applicable. Therefore, there are a maximum number of parameters at which an applicable solution was found for each isotherm. For example, if isotherms one and three were 
described by up to five parameters, but isotherm two was only described by up to four parameters, then the maximum number of parameters that can describe all isotherms is four.

Continuing from that same example, each of the four solutions (i.e., the solutions for one, two, three, and four parameters) for the three isotherms is associated with an objective function value $\left(F_{o b j}\right)$ from which the best solution can be determined, and therefore the corresponding number of parameters that is most suitable for all isotherms. If the best solutions for each isotherm are different (i.e., isotherms one and three are best described with three parameters, but isotherm two is best described with two parameters), then the weighted average of the number of parameters, rounded down to the nearest integer, is taken as the best solution for all isotherms.

If the maximum adsorption capacity $\left(q_{\max }\right)$ of a system is known, then there is no need for an additional procedure. However, this information may not always be available and such a case needs to be prepared for accordingly. First, the $q_{\max }$ is treated as an adjustable parameter and the procedures in Sections 3.2.2 through 3.2.4 would all still apply. Once the final solution is obtained, the optimized $q_{\max }$ parameters for each isotherm are compared alongside the respective euclidean norms of that solution. A weighted average of the $q_{\max }$ parameters is then taken and the entire procedure is rerun from the beginning to find a solution, which has the same $q_{\max }$ for each isotherm. 


\section{Results from the GSTA Code and Model with Various Data Sets}

\subsection{Water Vapor on Zeolite $3 A$}

The first set of results was developed to describe the equilibrium behavior between water vapor and zeolite 3A. Data for these isotherms were determined experimentally by Grace Davison [15], a manufacturer of zeolite, and presented in a graphical format that had to be digitized in order to formulate the appropriate input data. An open-source software called Plot Digitizer [16] was used to digitize the Grace Davison plots. This was a very large data set with many data points and many isotherms, so it served as a vigorous test of the ability of the codes to handle big data.

For this set of data, the $q_{\max }$ parameter was known $(22.8 \mathrm{~kg} / 100 \mathrm{~kg})$ [8] and was therefore given to the code as an input. Final results from the code are displayed in Fig. 2, which shows the digitized data points with the model results from the temperature dependent parameters shown on Table 2. The optimum number of equilibrium parameters to describe this system was found to be six $(m=6)$. However, it should be noted that the original authors of the GSTA model [8] had concluded that the best solution to this system required seven parameters $(m=7)$.

It was found that the both the maximum allowable parameter and smoothness criteria, mentioned in the Appendix and seen in Fig. A1, was the cause of the algorithms being unable to recreate the results given by the original authors of the model. By disabling these criteria, the code was able to find an applicable solution at seven parameters that was comparable to the solution given in literature [8] (see Table 2). Note that Llano-Restrepo and Mosquera [8] report their linear regression (Table 2$)$ only after eliminating an outlying data point $\left(K_{3}\right)$ at $60{ }^{\circ} \mathrm{C}$. The 
code reconstructed the same outlying data point that was observed by Llano-Restrepo and Mosquera [8] shown in Fig. 3. Without removing this point, the linear regression of the data to obtain the energy terms $\left(\Delta H_{n}{ }^{o}\right.$ and $\left.\Delta S_{n}{ }^{\circ}\right)$ is significantly and negatively impacted (see Table 2), but if that point were eliminated from the code's regression as well, then the results would essentially be the same.

To further investigate the effect of the $K_{3}$ parameter on this set of data, a sensitivity analysis was performed using the code's regression of that parameter without removing the outlier. The solid line in Fig. 4 shows the result of the 7-parameter GSTA temperature dependent model if the outlying point from Fig. 3 is not removed. What this demonstrates is that the first isotherm $(273.15 \mathrm{~K})$ is very sensitive to the $K_{3}$ parameter, while the other isotherms do not appear sensitive at all. To verify the 7-parameter model sensitivity, the results were recreated at seven parameters, but all $K_{3}$ parameter values were set to zero. The dotted line in Fig. 4 shows the result from this and confirms that while the first isotherm is sensitive to the value of $K_{3}$, all other isotherms are not. Because the majority of the model lacks sensitivity to $K_{3}$, it may be unnecessary to consider this parameter in the solution. However, this parameter cannot simply be thrown out just because it does not follow the linear trends in Fig. 3. Therefore, the 6-parameter solution obtained by the GSTA algorithms may be more applicable to this data than the 7parameter recorded in literature [8].

\section{2. $\mathrm{CO}_{2}, \mathrm{H}_{2} \mathrm{~S}$, and $\mathrm{C}_{3} \mathrm{H}_{8}$ on $\mathrm{H}$-mordenite}

The next series of equilibrium data employed in this study was published by Talu and Zwiebel [17], who had experimentally measured several gas-solid adsorption isotherms for $\mathrm{CO}_{2}$, 
$\mathrm{H}_{2} \mathrm{~S}$, and $\mathrm{C}_{3} \mathrm{H}_{8}$ on an H-mordenite adsorbent. Each adsorbate-adsorbent system displayed a slightly different equilibrium behavior and in no case was the maximum adsorbent capacity $\left(q_{\max }\right)$ known. Therefore, this set of data would test how well the code could estimate the capacity for each system and the versatility of the model to describe different behaviors.

Parameter results from the GSTA code are shown on Table 3 along with the correlation coefficients $\left(\mathrm{R}^{2}\right)$. The optimal number of parameters for each system was found to be six parameters for the $\mathrm{CO}_{2}$ isotherms and two parameters for the $\mathrm{H}_{2} \mathrm{~S}$ and $\mathrm{C}_{3} \mathrm{H}_{8}$ isotherms. Maximum adsorption capacities were optimized to approximately $2.88 \mathrm{mmol} / \mathrm{g}, 3.02 \mathrm{mmol} / \mathrm{g}$, and $1.48 \mathrm{mmol} / \mathrm{g}$ for $\mathrm{CO}_{2}, \mathrm{H}_{2} \mathrm{~S}$ and $\mathrm{C}_{3} \mathrm{H}_{8}$ isotherms, respectively. Fig. 5 through Fig. 7 show the results of the temperature dependent GSTA model using the parameters found on Table 3. Although Talu and Zwiebel [17] did not provide parameters for their own correlations with these single component isotherms, they did comment on the order of preferential adsorption: $\mathrm{H}_{2} \mathrm{~S}>$ $\mathrm{CO}_{2}>\mathrm{C}_{3} \mathrm{H}_{8}$. This behavior was replicated by model and reflected in the estimation of the $q_{\max }$ parameter.

\section{3. $\mathrm{CH}_{4}, \mathrm{CO}, \mathrm{CO}_{2}, \mathrm{H}_{2}$, and $\mathrm{H}_{2} \mathrm{~S}$ on Activated Carbon}

The final set of data examined was a series of five single-species systems on an activated carbon adsorbent. Isothermal data were measured experimentally by Ritter and Yang [7] and published along with their own correlated parameters for the data using a modified Langmuir isotherm model, which provided an empirical relationship between the Langmuir isotherm capacity $\left(q_{\max }\right)$ and temperature. All isotherms are reported at the high-pressure regimes ( 0.1 to 6.5 MPa), as opposed to the previous data tested in Sections 4.1 and 4.2, which were all low 
pressure. This will test the ability of the algorithms and model to adapt to high-pressure isothermal behaviors.

Table 4 shows the results for the energy terms found through the optimization of the GSTA model parameters with the equilibrium data. As on Tables 2 and 3, all parameters show a very orderly and well-behaved correlation across the temperature range. Interestingly, the code had found that the optimal number of parameters for all systems was one, which means that the GSTA model took the form of the Langmuir model, which Ritter and Yang [7] had used in their own correlations. However, a direct comparison between the GSTA parameters and the Ritter and Yang parameters was not possible due to some differences in utilization of the two models.

Recall in Section 1.1.2 that the derivation of the Langmuir model included four basic assumptions to be applied to the adsorbate-adsorbent system [1]. Of these four assumptions, statements (a) and (c) formulate the fundamental definition of the $q_{\max }$ parameter, which defines it as a constant for the particular adsorption system (i.e., it should not vary with temperature). This is how the $q_{\max }$ parameter is treated in the GSTA model, but not how Ritter and Yang [7] treated the parameter in their correlations. Ritter and Yang allowed the maximum capacity to vary with each isotherm and declared an empirical relationship between their obtained $q_{\max }$ and temperature.

Although a direct comparison of parameters is not possible, the relative "fitness" of each model can still be directly compared. Table 5 shows the average euclidean norms and errors between each model and the data for each isotherm. The Ritter and Yang [7] model was able to better describe the data than the original GSTA model overall, but it did so at the cost of more parameters. Because the $q_{\max }$ was allowed to vary with temperature, each isotherm of Ritter and Yang was described with four temperature parameters, two for $q_{\max }$ and two for $K$. On the other 
hand, the GSTA code found constant maximum capacities for each system and therefore could describe the data with only two temperature parameters, $\Delta H_{l}{ }^{o}$ and $\Delta S_{l}^{o}$.

In an attempt to recreate the Ritter and Yang [7] results, the code was temporarily modified to allow the $q_{\max }$ parameter to vary with temperature and then the code was rerun. Those results can also be viewed on Table 5 and are shown to be the best overall description of the data. However, this temporary modification to the GSTA model did not find the same empirical relationship that Ritter and Yang [7] had used to correlate the maximum capacities with temperature. This lack of any cohesive relationship between $q_{\max }$ and temperature is a good indication that no such relationship should actually exist for this type of isotherm model.

Other variations in these results could be explained by the different techniques used for obtaining the optimum parameters. Ritter and Yang used a linear least squares method [7] combined with the linearized form of the Langmuir model (see Table 1) to obtain the model parameters, while the GSTA code used a Levenberg-Marquardt non-linear optimization routine [14] to optimize the model parameters. Variations between the model parameters and overall description are a direct result of the transformation of the Langmuir model to a linear form [18]. When a model is transformed, or linearized, the relative error is also transformed which may result in a deviation between obtained and real solutions.

To gain a better understanding of the differences between the GSTA model and the Ritter and Yang [7] model, Fig. 8 through Fig. 12 show the temperature dependent GSTA model (solid line) and the temperature dependent Ritter and Yang [7] results (dotted line) against the experimental data recorded in literature. Between the pressure regions that contained data, the two models are very close to each other, but at the higher pressures the two models start to diverge. This is because the Ritter and Yang model will approach a different limit for each 
isotherm, while the GSTA model will approach the same limit. That limiting behavior is defined by the constant $q_{\max }$ parameters which the GSTA code had optimized for at approximately 5.88 $\mathrm{mmol} / \mathrm{g}, 5.15 \mathrm{mmol} / \mathrm{g}, 9.27 \mathrm{mmol} / \mathrm{g}, 4.59 \mathrm{mmol} / \mathrm{g}$, and $10.03 \mathrm{mmol} / \mathrm{g}$ for the $\mathrm{CH}_{4}, \mathrm{CO}, \mathrm{CO}_{2}, \mathrm{H}_{2}$, and $\mathrm{H}_{2} \mathrm{~S}$ isotherms, respectively.

\section{Discussion and Conclusions}

\subsection{Verification and Validation}

The verification of the GSTA modeling and optimization algorithms has been routinely performed throughout its development to ensure that no steps in its execution or routine utilized have been broken. Each different set of data, with additional test cases, was used to search out errors in code that may be revealed through the variability of the input that the code may be faced with. Although there may still be improvements to data handling routines that can be made, in the current version of the code no errors exist in any of the routines that are implemented.

Validation of the GSTA code was accomplished through the comparison of the results with those reported in Llano-Restrepo and Mosquera [8], which was the only known literature source that also used the GSTA model. Recall from Section 4.1 that the code, in its unaltered form, found a different solution than the solution in literature. However, the code was able to recreate the 7-parameter solution when some specific functionality of the code and the outlying $K_{3}$ point was neglected. Llano-Restrepo and Mosquera [8] had also found valid solutions between six and ten parameters, but did not report those parameters found. Their 7-parameter solution was chosen 
for two reasons: (a) it resulted in fewer oscillations in the isosteric heat of adsorption and (b) they could physically define a set of seven sites where water molecules could adsorb in a fully utilized zeolite.

However, it should be noted that the validity of the 7-parameter solution that Llano-Restrepo and Mosquera proposed [8] could only be attained by selectively disregarding an outlying data point to formulate the enthalpies and entropies associated with the third parameter $\left(K_{3}\right)$. Additionally, through the sensitivity analysis of the third parameter in Fig. 4 it is demonstrated that the 7-parameter model does not rely on all seven parameters in most cases. Only one of the isotherms $(273.15 \mathrm{~K})$ shows an affinity to that parameter, while all others are nearly unaffected by its magnitude.

Furthermore, Llano-Restrepo and Mosquera [8] acknowledge that the 7-parameter solution represents only a maximum plausible number of sites for the adsorbate-adsorbent system. It is therefore possible that the actual number of sites be lower than this maximum, which may imply that certain sites within the adsorbent matrix are unavailable or unattainable by the adsorbate molecules. Such restrictions could be due to space or diffusion limitations. In any case, the 6parameter solution obtained from the GSTA code developed here could therefore also be valid and possibly more significant due to the lack of sensitivity observed in the 7-parameter solution.

\subsection{Versatility and Reliability}

Through the results obtained in Section 4, it has been demonstrated that the developed algorithms can accommodate a wide range of variability in the adsorption behavior. This is likely due to the versatility of the GSTA model itself. Since the GSTA model contains an adjustable 
number of parameters, it could be utilized in describing any number of systems. Furthermore, the optimal number of parameters obtained could potentially reveal information regarding the adsorbate-adsorbent system, such as heterogeneity.

Recall that in 2.1 the GSTA model could be derived from the Heterogeneous Langmuir model and consider that the code was designed to find the optimal value for the number of parameters $(m)$. In the case in which the optimal number of parameters was found to be one, the GSTA model would take the form of the Langmuir model (i.e., a homogeneous adsorption model). For all other cases, the GSTA model would be a special case of the Heterogeneous Langmuir model. The $m$ parameter yields information as to the heterogeneity of the system (i.e., larger $m$ is a more heterogeneous system, while a smaller $m$ is more homogeneous). Therefore, the GSTA model would be valid for both heterogeneous and homogeneous isothermal data because it has the versatility to fit in both situations.

Additionally, both the Langmuir and Heterogeneous Langmuir models have been widely used and accepted models for describing both physical and chemical adsorption [1]. Having made the connection between the GSTA model and those isotherm models it would be reasonable to conclude that the GSTA model could also be used to describe both physical and chemical adsorption. However, this may have an impact on the physical interpretation of the parameters of the model.

All of the results obtained in Section 4 articulate the reliability of the GSTA model and the code developed for it. Recall the correlation coefficients $\left(\mathrm{R}^{2}\right)$ for the enthalpies and entropies on Tables 2, 3, and 4. Every parameter optimized was found to have a nearly perfect linear relationship with temperature as evident from all $\mathrm{R}^{2}$ values being 0.9920 or better. The accuracy of these correlations dictates the ability of the model to reliably predict the adsorption behavior 
between the applicable ranges of temperature. This feature is especially important if the model is to be applied to real systems in which isothermal conditions are rarely achieved.

\subsection{Extensions and Improvements}

As discussed in Section 1.2.1, in order to evaluate the spreading pressure of an adsorption system, the isotherm model must display linear behavior at low pressure $[1,6]$. To extend the GSTA model into other models, such as the Ideal Adsorbed Solution Theory (IAST) for predicting multicomponent behavior of gas mixtures, the spreading pressure must be accurately evaluated [6,13]. Llano-Restrepo and Mosquera also recognized the need for this Henry's Law like behavior and demonstrated themselves that the GSTA isotherm does have a finite limit at very low pressure [8]. This would be logical since the GSTA isotherm behaves similarly to the Langmuir model.

Unfortunately, this also means that the GSTA model is thermodynamically inconsistent with the Gibbs' expression for gas-solid adsorption, as there is a finite limit of $q_{\max }$ as the pressure increases infinitely [2]. However, it may be possible to formulate a new, modified version of the GSTA model, which is thermodynamically consistent. There is a two-step procedure discussed by Tóth [2] in which inconsistent isotherms can be modified through a general mathematical approach. This method has been used before to derive a three-parameter modified Langmuir model in which the monolayer capacity is achievable at a given saturation pressure.

Additionally, this inconsistent behavior of the GSTA model may account for some of the errors and divergence observed in the results obtained for the Ritter and Yang [7] data in Section 4.3. Since all of these isotherms are measured and analyzed at high pressures, the GSTA and 
Langmuir models may not be the most applicable equilibrium models to describe that set of data. Although the data were described reasonably well, discrepancies observed could be the result of going beyond the actual monolayer capacity of the adsorbent material. Therefore, while the GSTA model may be general enough to be utilized for nearly any low to mid-range pressure systems, modifications may be necessary in order for results to be considered significant and consistent for higher-pressure systems.

In conclusion, the GSTA model proposed by Llano-Restrepo and Mosquera [8] and the code developed in this paper have performed admirably for the variety of systems tested. Each different adsorbate-adsorbent isotherm was described with high accuracy and the largest of the errors were observed only in the highest-pressure ranges. All solutions obtained showed a wellbehaved linear behavior with temperature, allowing predictions to be reliably made for the temperature for which data were not available. Although there were many complications with using the GSTA model, the culmination of careful programming with today's modern computing languages have made the utilization of such a complex adsorption model not only feasible, but also robust and reliable as well.

\section{Acknowledgements}

This research is being performed using funding received from the DOE Office of Nuclear Energy's Nuclear Energy University Programs and is conducted at the Georgia Institute of Technology in collaboration with the Oak Ridge National Laboratory (ORNL). ORNL is managed by UT-Battelle, LLC under Contract DE-AC05-00OR22725 with the U.S. Department of Energy. 


\section{Appendix}

\section{Code Development and Structure}

A code was developed in $\mathrm{C} / \mathrm{C}++$ in order to apply all of the techniques discussed in Section 3.2 and to handle the variability that may be observed in the various input data, such as data set sizes and parameter units. Using dynamic memory allocation objects in $\mathrm{C}++$ allows the code to handle any number of isotherms and any number of data points within each isotherm set. The code is also completely agnostic of the units of adsorption capacity; this means that whatever units of capacity are given will be the same units that come out from the algorithms. However, the gas phase concentrations of the adsorbate must be given in $\mathrm{kPa}$. This is because the dimensionless equilibrium parameters $\left(K_{n}{ }^{o}\right)$ of the GSTA model are calculated by Eq. (3), which uses $100 \mathrm{kPa}$ as standard state pressure $\left(P^{o}\right)$.

The optimization routine for finding the parameters of the GSTA model was accomplished by using a Levenberg-Marquardt non-linear least squares algorithm written in C by Wuttke [14]. Developed around that routine is the $\mathrm{C} / \mathrm{C}++$ code, which handles input/output and the various functions and data structures necessary to deal with the complications discussed in Section 3.2 above. A flowchart outlining the major code structure is provided in Fig. A1.

The main features of the code are in the three loops that make up the bulk of its structure. Each loop will be executed at least once and then terminate when certain criteria are met. The inner most loop proceeds through each number of plausible parameters (n_par) for which the GSTA model may be valid, while the middle loop continues the solution searches for each 
number of isotherms (n_iso) that was given on input. For instances in which the value of the $q_{\max }$ parameter is unknown, the outer most loop will be executed twice in order to make a single estimate of this parameter for all isotherms. If $q_{\max }$ was given on input, then the loop is executed only once. The Boolean expression QMAX is used to determine whether or not the parameter was given on input.

Within the inner loop, there are three criteria that may force an early termination of the loop. These are put in place to reduce the total number of evaluations by eliminating unnecessary solution searches. The first criterion checks whether or not the current data set being evaluated is the first isotherm (iso $=0$ ) given on input. If it is not, then it checks to see how many parameters the previous isotherm was allowed to have $(\max )$. There is no need to run the optimization for a number of parameters greater than the maximum encountered, therefore the loop would terminate early when $\mathrm{n} \_$par is greater than max.

The next two criteria look at the obtained solution after the optimization has been completed. If the solution vector contains "noise" (i.e., the solution is non-smooth), then those results are kicked out and the loop is terminated. Also, if the euclidean norm of the current solution were not improving from previous solutions, then the loop would also be terminated. The culmination of these criteria has reduced the total evaluations needed to reach a final solution by up to $80 \%$. 


\section{References}

[1] C. Tien, Adsorption Calculations and Modeling, Butterworth-Heinemann, Newton, MA, 1994.

[2] J. Tóth (Ed.), Adsorption Theory Modeling and Analysis, Marcel Dekker, Inc. New York, NY, 2002.

[3] J. Tóth, Adv. Colloid Interf. Sci. 55 (1995) 1-239.

[4] D.P. Valenzuela, A.L. Myers, Adsorption Equilibrium Handbook, Prentice-Hall, Englewood Cliffs, NJ, 1989.

[5] A.L. Myers, AIChE J. 29 (1983) 691-693.

[6] O. Talu, A.L. Myers, AIChE J. 34 (1988) 1887-1893.

[7] J.A. Ritter, R.T. Yang, Ind. Eng. Chem. Res. 26 (1987) 1679-1686.

[8] M. Llano-Restrepo, M.A. Mosquera, Fluid Phase Equilibria, 283 (2009) 73-88.

[9] H. Moon, C. Tien, Chem. Eng. Sci. 43 (1988) 2967-2980.

[10] R.P. Grimaldi, Discrete and Combinatorial Mathematics, Pearson Education, Inc. New York, NY, 2004.

[11] K. Madsen, H.B. Nielsen, O. Tingleff, Methods for Non-Linear Least Squares Problems, Informatics and Mathematical Modeling, Technical University of Denmark, 2004.

[12] R.M.A. Roque-Malherbe, Adsorption and Diffusion in Nonporous Materials, CRC Press, Taylor \& Francis Group, Boca Raton, FL, 2007.

[13] A.L. Myers, J.M. Prausnitz, AIChE J. 11 (1965) 121-127.

[14] J. Wuttke, Version <lmfit-3.4>, <http://joachimwuttke.de/lmfit>, January 21, 2013 
[15] W.R. Grace \& Co. Grace Davison: Sylobead Adsorbents for Process Applications, Columbia, MD, 2002.

[16] <http://plotdigitizer.sourceforge.net/>, October 16, 2013.

[17] O. Talu, I. Zwiebel, AIChE J. 32 (1986) 1263-1276.

[18] D.G. Kinniburgh, ES\&T, 20 (1986) 895-904.

\section{Figure Captions}

Fig. 1. Visualization of the Educated Initial Value Guessing procedure used by the GSTA code to estimate the initial values of the parameters. A good estimate is needed to ensure that the nonlinear solution algorithm optimizes the parameters appropriately and can reliably find a solution. By using information gained from previously optimized sets of parameters, a more educated guess can be made for the next set.

Fig. 2. Equilibrium adsorption isotherms of water vapor on zeolite $3 \mathrm{~A}$ at various temperatures. The symbols represent experimental data points which were digitized while the solid lines show the result of the GSTA model using the temperature dependent equilibrium parameters formulated from the enthalpies and entropies reported on Table 2 for the 6-parameter solution. 
Fig. 3. Relationship between the dimensionless equilibrium constants of the GSTA isotherm and the system temperature for the 7-parameter solution to the water vapor and zeolite equilibrium data. The outlying data point for the third equilibrium point $(n=3)$ is circled and its influence on the linear interpolation is shown by the solid line. Correlation coefficients for all the linear regressions can be viewed on Table 2 under the Modified Code header.

Fig. 4. Equilibrium adsorption isotherms of water vapor on zeolite $3 \mathrm{~A}$ at various temperatures. The symbols represent the experimental data points and the solid lines show the result of the GSTA model using the temperature dependent parameters formulated from the enthalpies and entropies reported on Table 2 for the 7-parameter solution found under the Modified Code header. The dotted line shows the result of the GSTA model using those same parameters, except the third parameter $\left(K_{3}\right)$ is replaced with zeros for all isotherms. This shows that the first isotherm $(273.15 \mathrm{~K})$ is very sensitive, while all others are barely, or not at all, sensitive to the third parameter.

Fig. 5. Equilibrium adsorption isotherms of $\mathrm{CO}_{2}$ on $\mathrm{H}$-mordenite at various temperatures. The symbols represent the experimental data points and the solid lines show the results of the GSTA model using the temperature dependent parameters formulated from the enthalpies and entropies reported on Table 3. 
Fig. 6. Equilibrium adsorption isotherms of $\mathrm{H}_{2} \mathrm{~S}$ on $\mathrm{H}$-mordenite at various temperatures. The symbols represent the experimental data points and the solid lines show the results of the GSTA model using the temperature dependent parameters formulated from the enthalpies and entropies reported on Table 3.

Fig. 7. Equilibrium adsorption isotherms of $\mathrm{C}_{3} \mathrm{H}_{8}$ on $\mathrm{H}$-mordenite at various temperatures. The symbols represent the experimental data points and the solid lines show the results of the GSTA model using the temperature dependent parameters formulated from the enthalpies and entropies reported on Table 3.

Fig. 8. Equilibrium adsorption isotherms of $\mathrm{CH}_{4}$ on activated carbon at various temperatures. The symbols represent the experimental data points and the solid and dashed lines show the GSTA and Ritter and Yang [7] model results, respectively, using the temperature dependent parameters formulated from the enthalpies and entropies reported on Table 4.

Fig. 9. Equilibrium adsorption isotherms of $\mathrm{CO}$ on activated carbon at various temperatures. The symbols represent the experimental data points and the solid and dashed lines show the GSTA and Ritter and Yang [7] model results, respectively, using the temperature dependent parameters formulated from the enthalpies and entropies reported on Table 4. 
Fig. 10. Equilibrium adsorption isotherms of $\mathrm{CO}_{2}$ on activated carbon at various temperatures. The symbols represent the experimental data points and the solid and dashed lines show the GSTA and Ritter and Yang [7] model results, respectively, using the temperature dependent parameters formulated from the enthalpies and entropies reported on Table 4.

Fig. 11. Equilibrium adsorption isotherms of $\mathrm{H}_{2}$ on activated carbon at various temperatures. The symbols represent the experimental data points and the solid and dashed lines show the GSTA and Ritter and Yang [7] model results, respectively, using the temperature dependent parameters formulated from the enthalpies and entropies reported on Table 4.

Fig. 12. Equilibrium adsorption isotherms of $\mathrm{H}_{2} \mathrm{~S}$ on activated carbon at various temperatures. The symbols represent the experimental data points and the solid and dashed lines show the GSTA and Ritter and Yang [7] model results, respectively, using the temperature dependent parameters formulated from the enthalpies and entropies reported on Table 4.

Fig. A1. Flowchart for the main functions within the GSTA optimization code. The logical path of the code flows down from the "Start" and follows the arrows. At each branch, a decision has to be made which determines the next process and/or the "Breaks" necessary to exit loops and reach the "End" of the program. 
Table 1

A Summary of Common Adsorption Models

\begin{tabular}{llll}
\hline Model & Equation & Linear Form & Parameters \\
\hline Freundlich & $q=K_{F} p^{\mathbf{1 / a}}$ & $\log (q)=\log \left(K_{F}\right)+\frac{\mathbf{1}}{a} \log (p)$ & $K_{F}, a$ \\
Langmuir & $q=\frac{q_{\max } K_{L} p}{\mathbf{1}+K_{L} p}$ & $\frac{\mathbf{1}}{q}=\frac{\mathbf{1}}{q_{\max } K_{L}} \frac{\mathbf{1}}{p}+\frac{\mathbf{1}}{q_{\max }}$ & $K_{L} q_{\max }$ \\
Tóth & $q=\frac{q_{\max } K_{T} p}{\left(\mathbf{1}+\left(K_{T} p\right)^{b}\right)^{\mathbf{1 / b}}}$ & None & $K_{T}, q_{\max }, b$ \\
$\begin{array}{l}\text { Heterogeneous } \\
\text { Langmuir }\end{array}$ & $q=\sum_{n=\mathbf{1}}^{m} \frac{q_{n} K_{L, n} p}{\mathbf{1}+K_{L, n} p}$ & None & $K_{L, 1}, K_{L, 2}, \ldots K_{L, m}$ \\
\hline
\end{tabular}

\section{Table 2}

Comparison of the relevant energy terms for the water vapor isotherms optimized at six and seven parameters. The units of $\Delta H_{n}{ }^{o}$ and $\Delta S_{n}{ }^{o}$ are $\mathrm{kJ} / \mathrm{mol}$ and $\mathrm{J} /(\mathrm{K} \mathrm{mol})$, respectively.

\begin{tabular}{cccccccccc}
\hline & \multicolumn{3}{c}{ Our Code Results } & \multicolumn{3}{c}{ Modified Code Results } & \multicolumn{3}{c}{ Llano-Restrepo \& Mosquera [8] } \\
& $\Delta H_{n}{ }^{\circ}$ & $\Delta S_{n}{ }^{\circ}$ & $\mathrm{R}^{2}$ & $\Delta H_{n}{ }^{o}$ & $\Delta S_{n}{ }^{\circ}$ & $\mathrm{R}^{2}$ & $\Delta H_{n}{ }^{\circ}$ & $\Delta S_{n}{ }^{o}$ & $\mathrm{R}^{2}$ \\
\hline 1 & -63.5 & -97.3 & 0.9979 & -64.8 & -99.6 & 0.9975 & -64.7 & -98.0 & 0.9977 \\
2 & -120.6 & -204.2 & 0.9996 & -121.4 & -197.9 & 0.9991 & -122.0 & -198.0 & 0.9992 \\
3 & -184.5 & -339.5 & 0.9941 & -321.0 & -783.8 & 0.5395 & $-187.8^{\mathrm{a}}$ & $-342.0^{\mathrm{a}}$ & $0.9999^{\mathrm{a}}$ \\
4 & -233.4 & -423.0 & 0.9994 & -236.1 & -421.4 & 0.9980 & -236.0 & -420.0 & 0.9983 \\
5 & -287.9 & -548.1 & 0.9996 & -292.7 & -539.9 & 0.9996 & -292.5 & -535.0 & 0.9996 \\
6 & -329.6 & -653.3 & 0.9998 & -343.5 & -657.2 & 0.9995 & -343.8 & -654.0 & 0.9995 \\
7 & --- & --- & --- & -385.2 & -764.3 & 0.9998 & -385.7 & -763.0 & 0.9998 \\
\hline
\end{tabular}

${ }^{\mathrm{a}}$ The correlation reported in literature was obtained by disregarding the value of $K_{3}{ }^{o}$ which lay outside of the of the linear regression (see Fig. 3). 


\section{Table 3}

Relevant energy terms for the gas phase adsorption isotherms on an H-mordenite adsorbent. The units of $\Delta H_{n}^{o}$ and $\Delta S_{n}{ }^{o}$ are $\mathrm{kJ} / \mathrm{mol}$ and $\mathrm{J} /(\mathrm{K} \mathrm{mol})$, respectively.

\begin{tabular}{ccccc}
\hline $\begin{array}{c}\text { Gas } \\
\text { Component }\end{array}$ & $n$ & $\Delta H_{n}{ }^{o}$ & $\Delta S_{n}{ }^{o}$ & $\mathrm{R}^{2}$ \\
\hline & 1 & -36.67 & -76.86 & 0.9999 \\
& 2 & -82.98 & -207.66 & 1.0000 \\
$\mathrm{CO}_{2}$ & 3 & -105.44 & -266.22 & 0.9971 \\
& 4 & -145.36 & -391.34 & 0.9997 \\
& 5 & -169.19 & -474.27 & 0.9935 \\
& 6 & -195.59 & -558.59 & 0.9998 \\
\hline \multirow{2}{*}{$\mathrm{H}_{2} \mathrm{~S}$} & 1 & -34.30 & -67.57 & 0.9956 \\
& 2 & -67.15 & -164.09 & 0.9995 \\
\hline \multirow{2}{*}{$\mathrm{C}_{3} \mathrm{H}_{8}$} & 1 & -39.24 & -87.05 & 0.9969 \\
& 2 & -69.36 & -185.83 & 0.9990 \\
\hline
\end{tabular}

\section{Table 4}

Relevant energy terms for the gas phase adsorption isotherms on an Activated Carbon adsorbent. The units of $\Delta H_{n}{ }^{o}$ and $\Delta S_{n}{ }^{o}$ are $\mathrm{kJ} / \mathrm{mol}$ and $\mathrm{J} /(\mathrm{K} \mathrm{mol})$, respectively.

\begin{tabular}{ccccc}
\hline Gas Component & $n$ & $\Delta H_{n}{ }^{o}$ & $\Delta S_{n}{ }^{o}$ & $\mathrm{R}^{2}$ \\
\hline $\mathrm{CH}_{4}$ & 1 & -20.35 & -81.84 & 0.9962 \\
$\mathrm{CO}$ & 1 & -16.66 & -74.48 & 0.9965 \\
$\mathrm{CO}_{2}$ & 1 & -22.56 & -85.10 & 0.9962 \\
$\mathrm{H}_{2}$ & 1 & -13.42 & -84.89 & 0.9920 \\
$\mathrm{H}_{2} \mathrm{~S}$ & 1 & -20.64 & -72.61 & 1.0000 \\
\hline
\end{tabular}


Table 5

Euclidean Norms and Error Percentages for Gas-Solid Isotherms on Activated Carbon

\begin{tabular}{|c|c|c|c|c|c|c|c|}
\hline \multirow{2}{*}{$\begin{array}{c}\text { Gas } \\
\text { Component }\end{array}$} & \multirow{2}{*}{$T(\mathrm{~K})$} & \multicolumn{2}{|c|}{ Ritter and Yang [7] } & \multicolumn{2}{|c|}{ Original GSTA } & \multicolumn{2}{|c|}{ Modified GSTA } \\
\hline & & Norm & Error \% & Norm & Error \% & Norm & Error \% \\
\hline \multirow{3}{*}{$\mathrm{CH}_{4}$} & 296 & 0.38 & 2.39 & 0.38 & 3.43 & 0.10 & 0.65 \\
\hline & 373 & 0.38 & 4.68 & 0.63 & 9.28 & 0.11 & 1.62 \\
\hline & 480 & 0.12 & 4.17 & 0.26 & 7.57 & 0.06 & 2.81 \\
\hline \multirow{3}{*}{$\mathrm{CO}$} & 296 & 0.22 & 4.21 & 0.40 & 4.51 & 0.20 & 4.00 \\
\hline & 373 & 0.29 & 7.45 & 0.12 & 4.60 & 0.04 & 1.80 \\
\hline & 473 & 0.10 & 4.77 & 0.14 & 9.24 & 0.02 & 1.74 \\
\hline \multirow{3}{*}{$\mathrm{CO}_{2}$} & 296 & 0.32 & 3.25 & 0.42 & 2.63 & 0.25 & 2.51 \\
\hline & 373 & 0.58 & 7.65 & 0.52 & 11.40 & 0.12 & 2.69 \\
\hline & 480 & 0.08 & 3.19 & 0.24 & 11.72 & 0.06 & 2.93 \\
\hline \multirow{3}{*}{$\mathrm{H}_{2}$} & 296 & 0.17 & 6.99 & 0.17 & 9.41 & 0.16 & 7.92 \\
\hline & 373 & 0.03 & 1.95 & 0.02 & 1.64 & 0.01 & 0.88 \\
\hline & 480 & 0.03 & 5.51 & 0.03 & 4.16 & 0.03 & 6.14 \\
\hline \multirow{3}{*}{$\mathrm{H}_{2} \mathrm{~S}$} & 296 & 0.30 & 1.85 & 0.74 & 5.15 & 0.30 & 2.01 \\
\hline & 373 & 0.16 & 1.93 & 0.31 & 4.78 & 0.10 & 1.57 \\
\hline & 480 & 0.07 & 1.67 & 0.19 & 6.97 & 0.03 & 1.25 \\
\hline Averages & & 0.22 & 4.11 & 0.30 & 6.43 & 0.11 & 2.70 \\
\hline
\end{tabular}




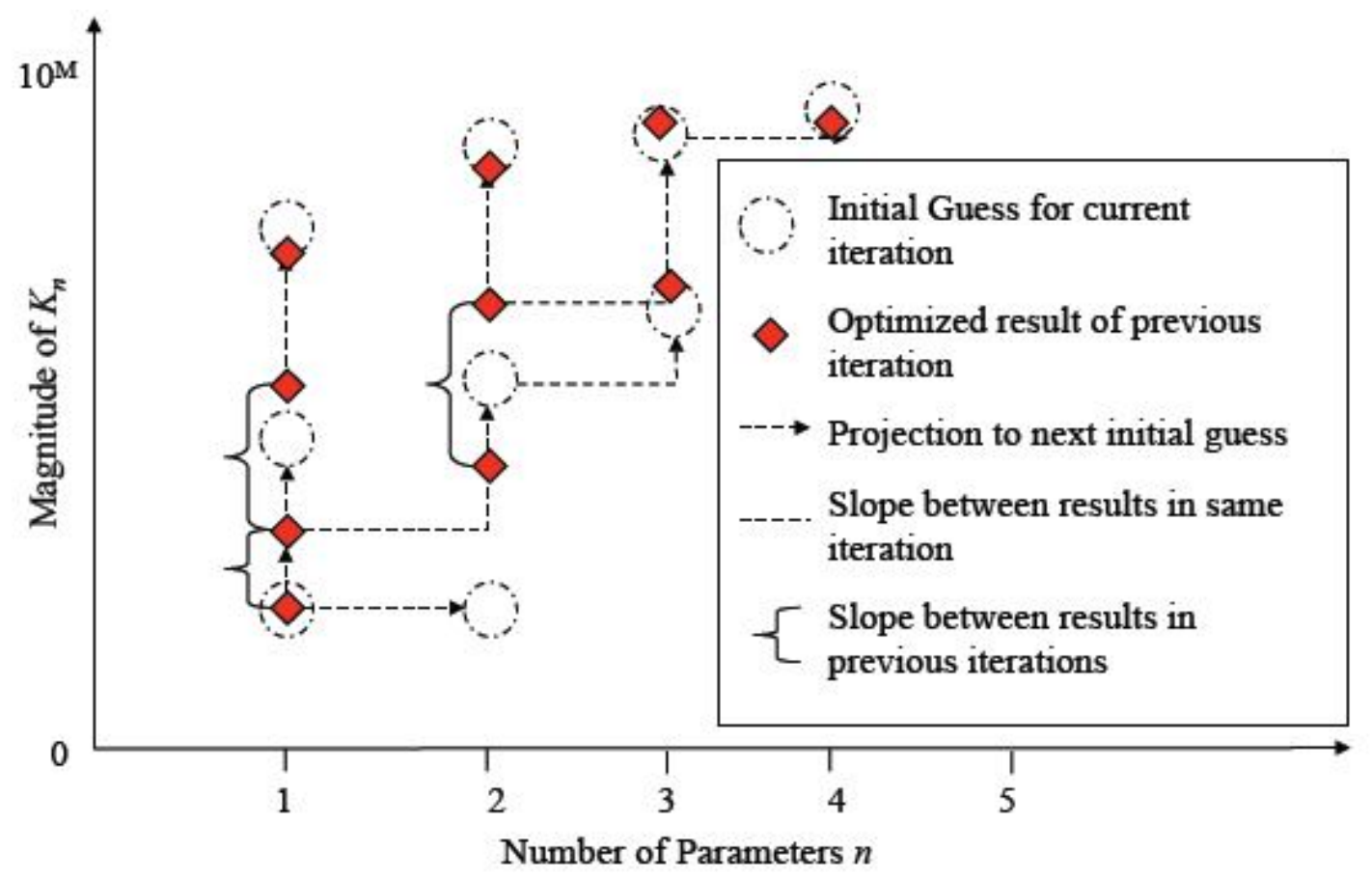

Fig. 1 


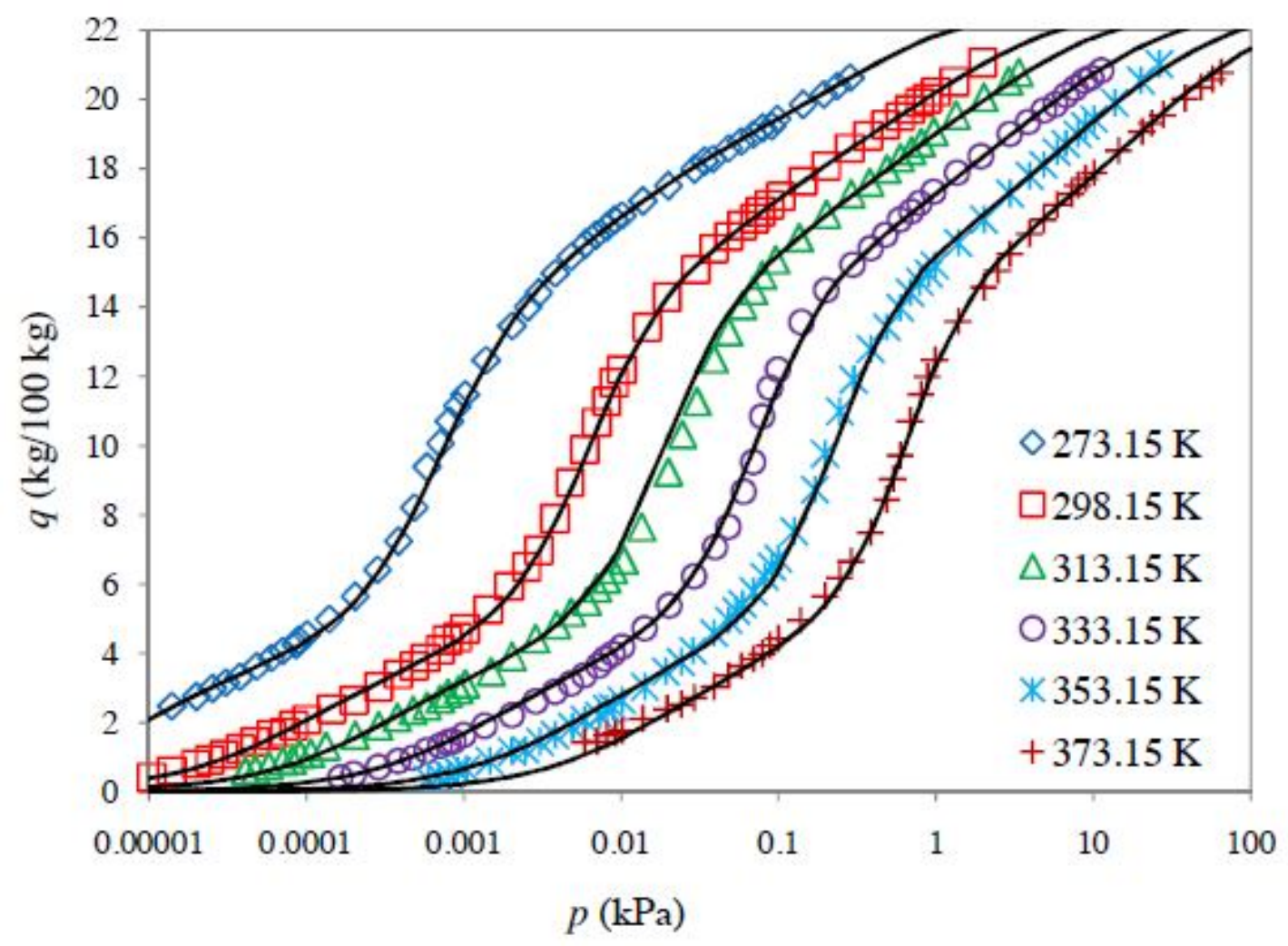

Fig. 2 


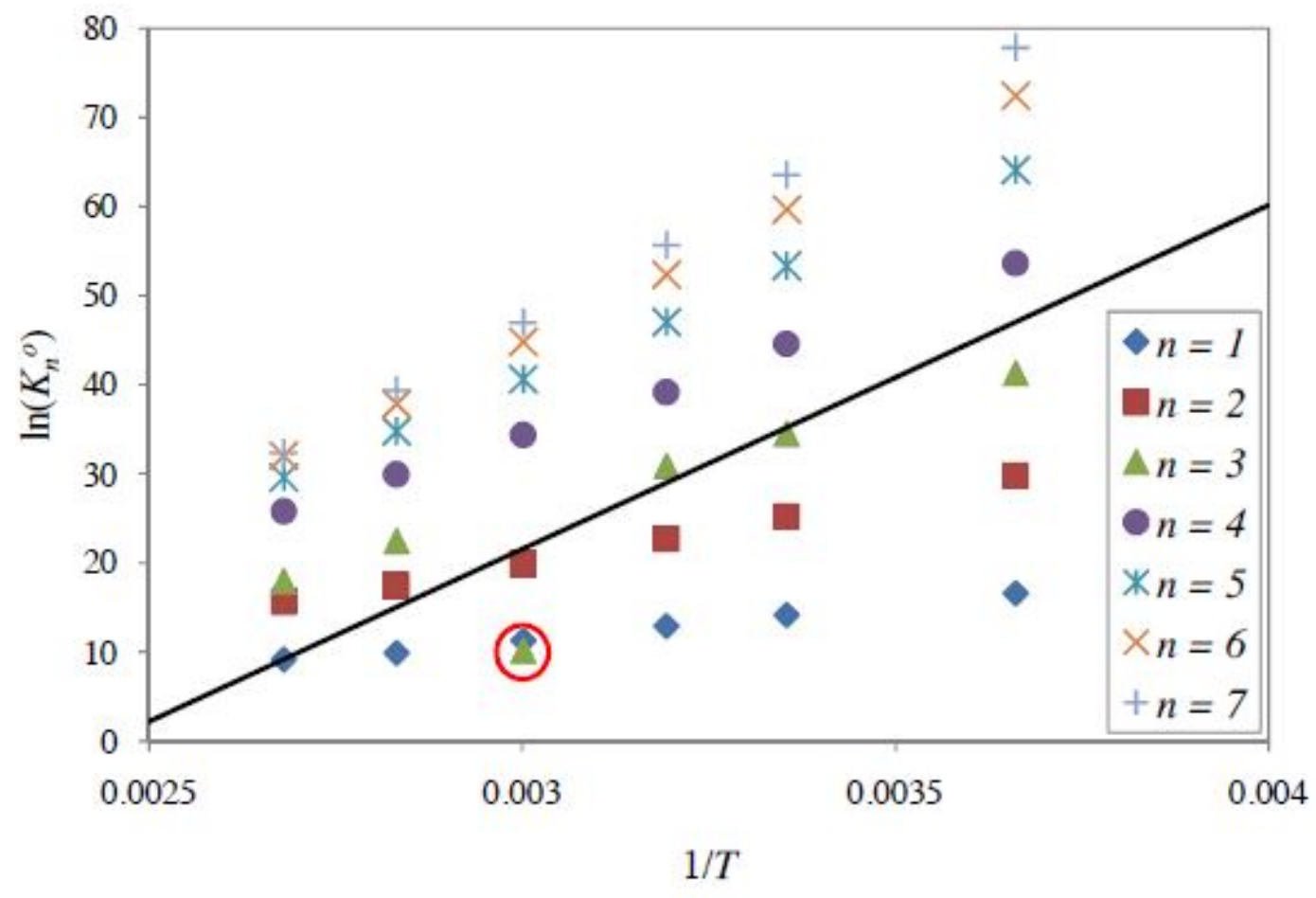

Fig. 3 


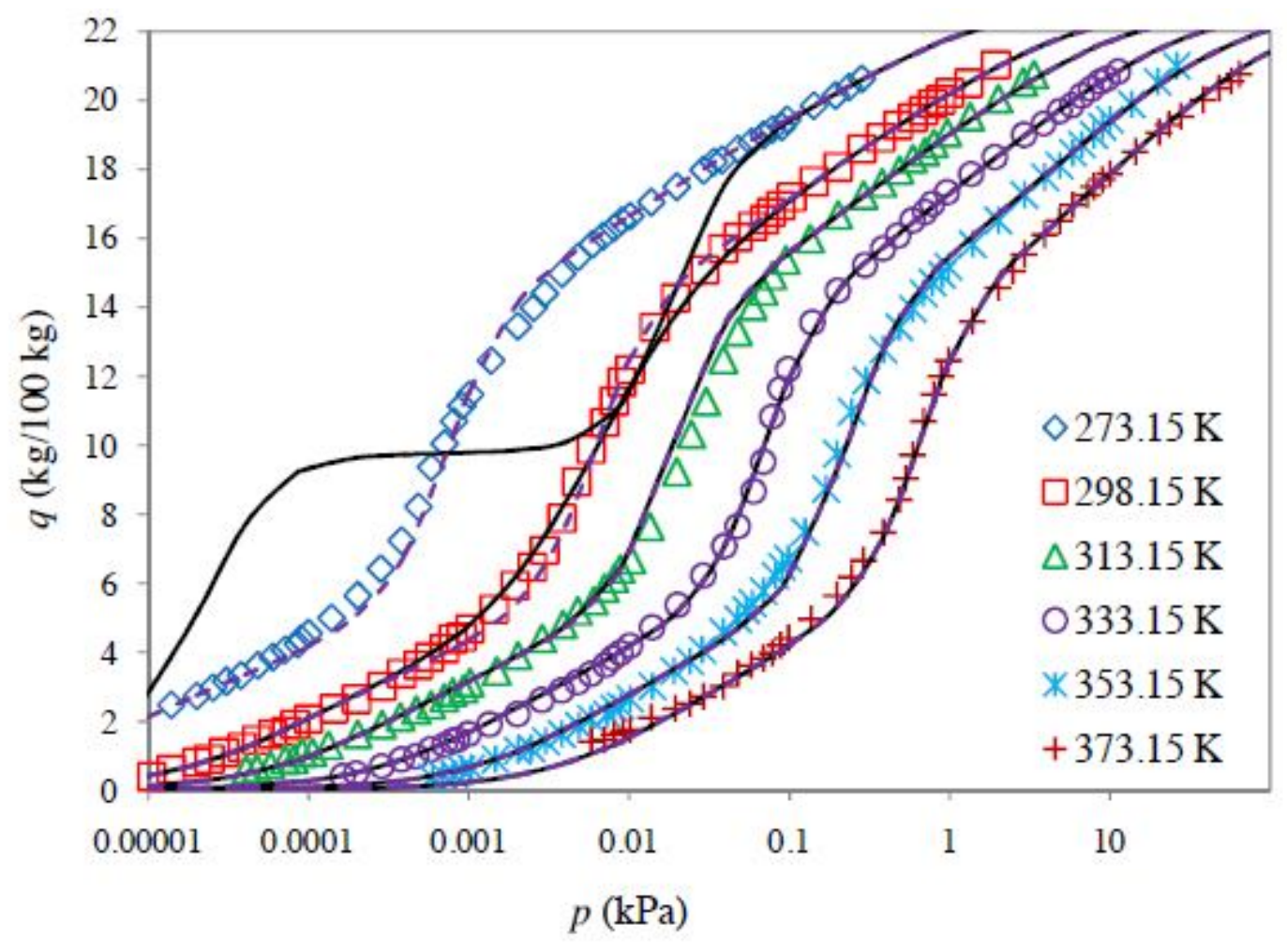

Fig. 4 


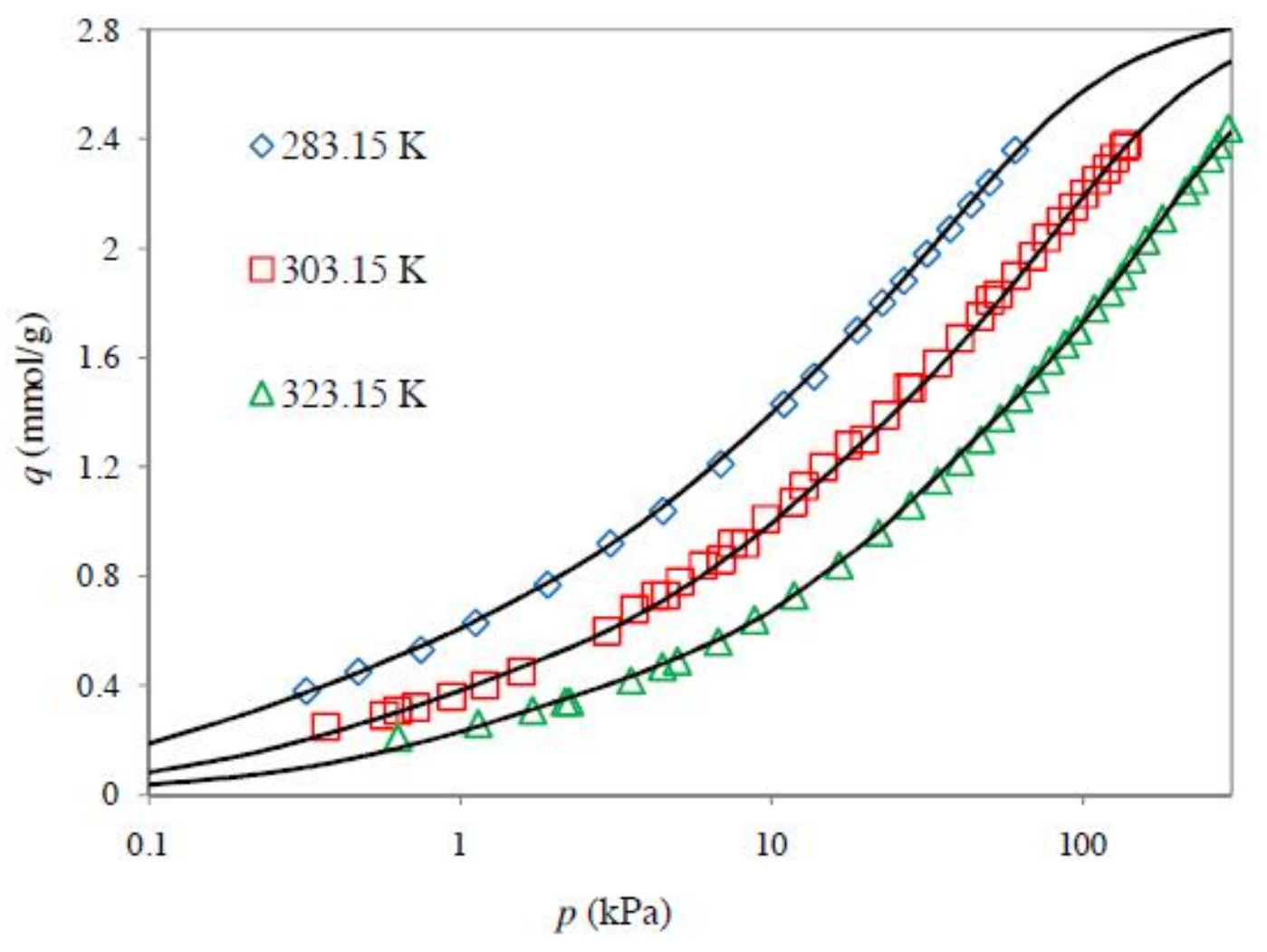

Fig. 5 


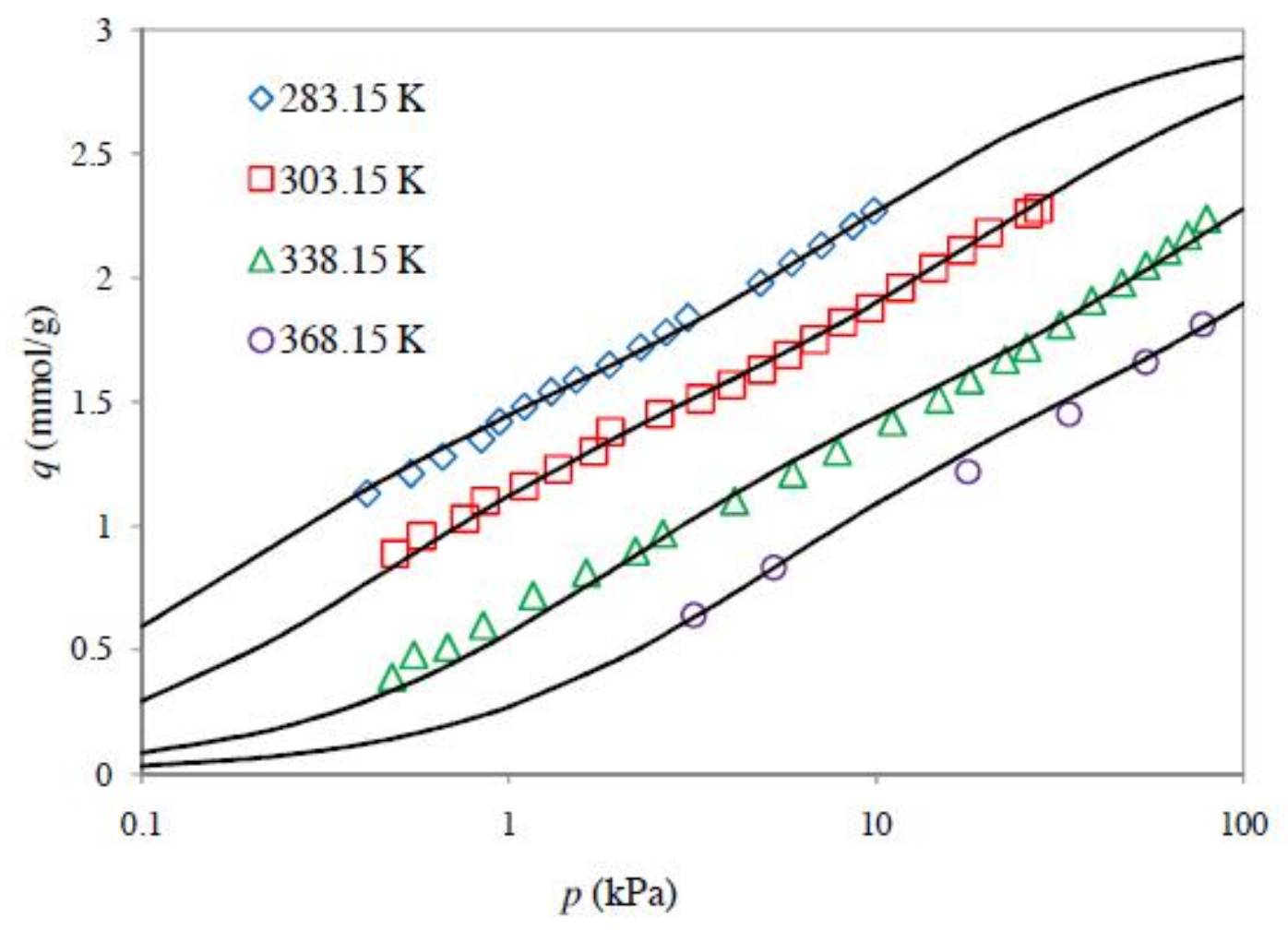

Fig. 6 


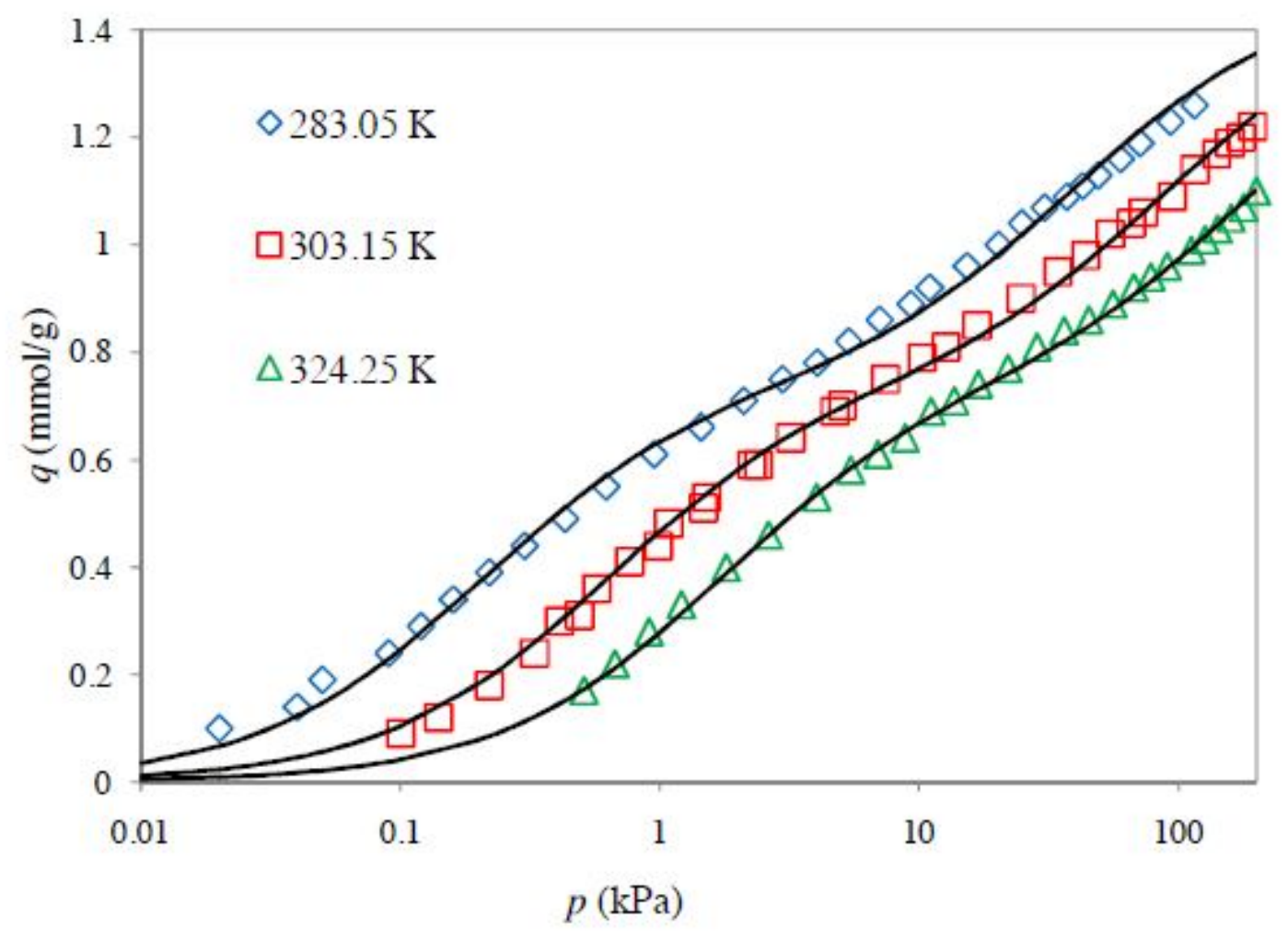

Fig. 7 


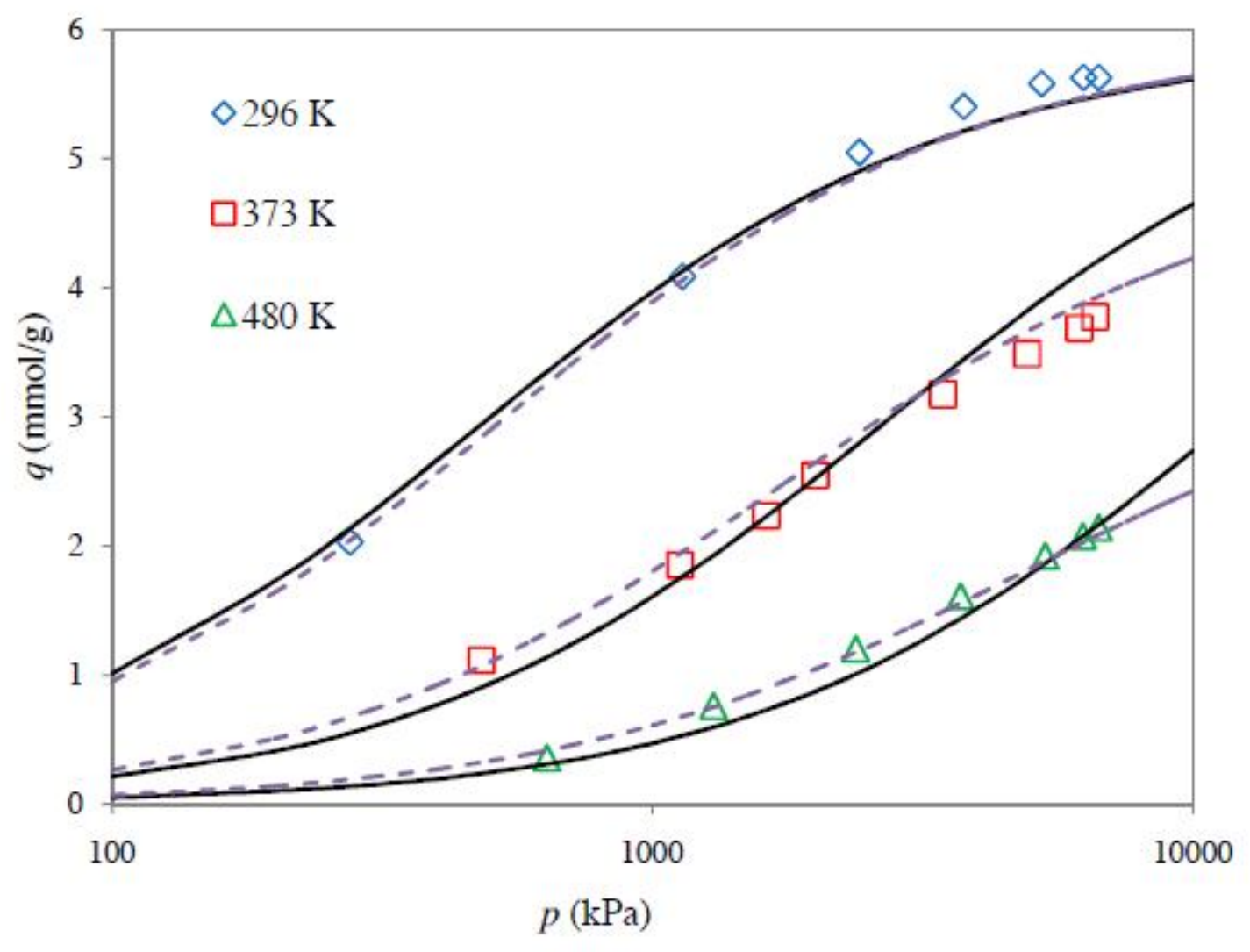

Fig. 8 


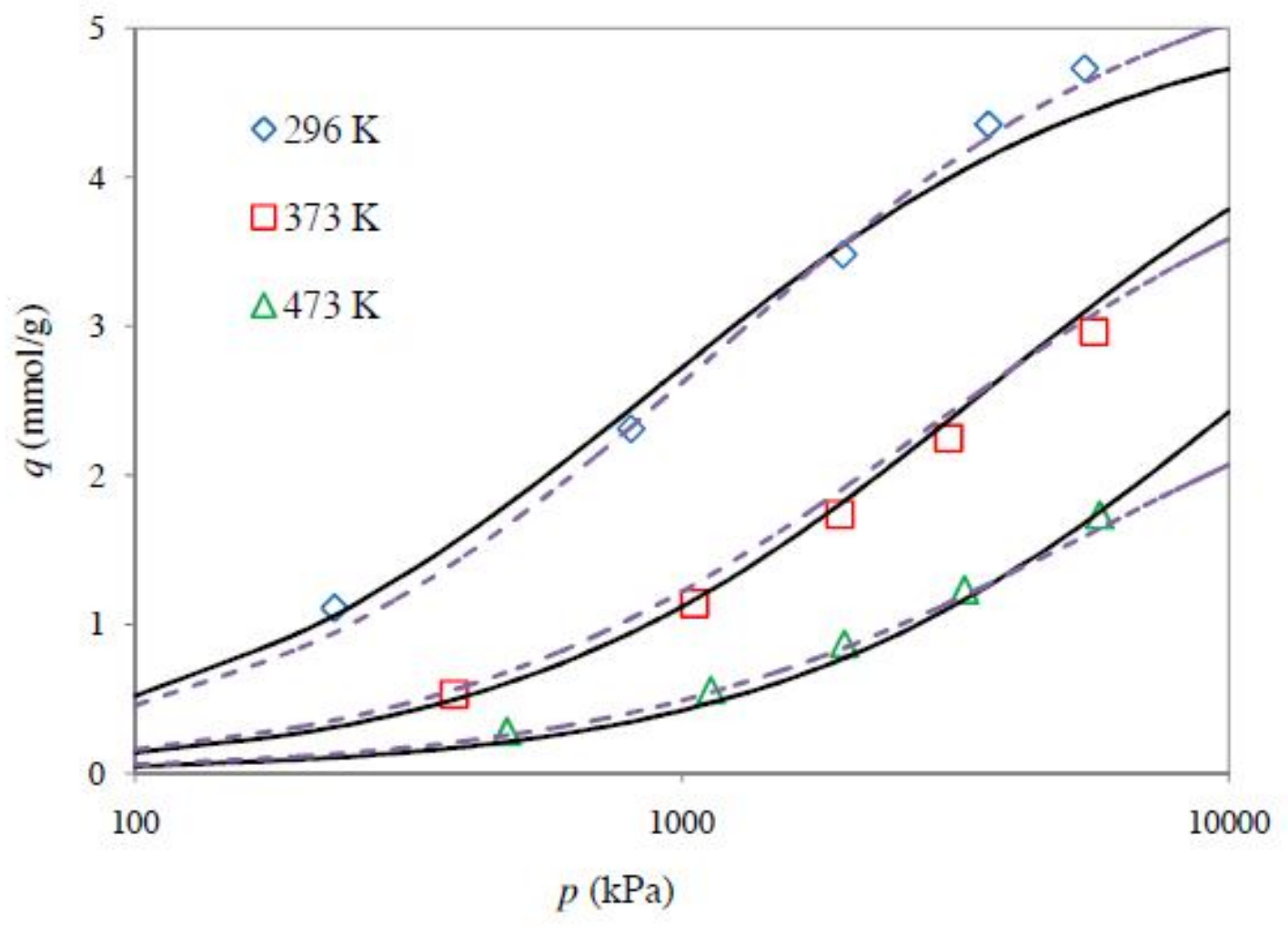

Fig. 9 


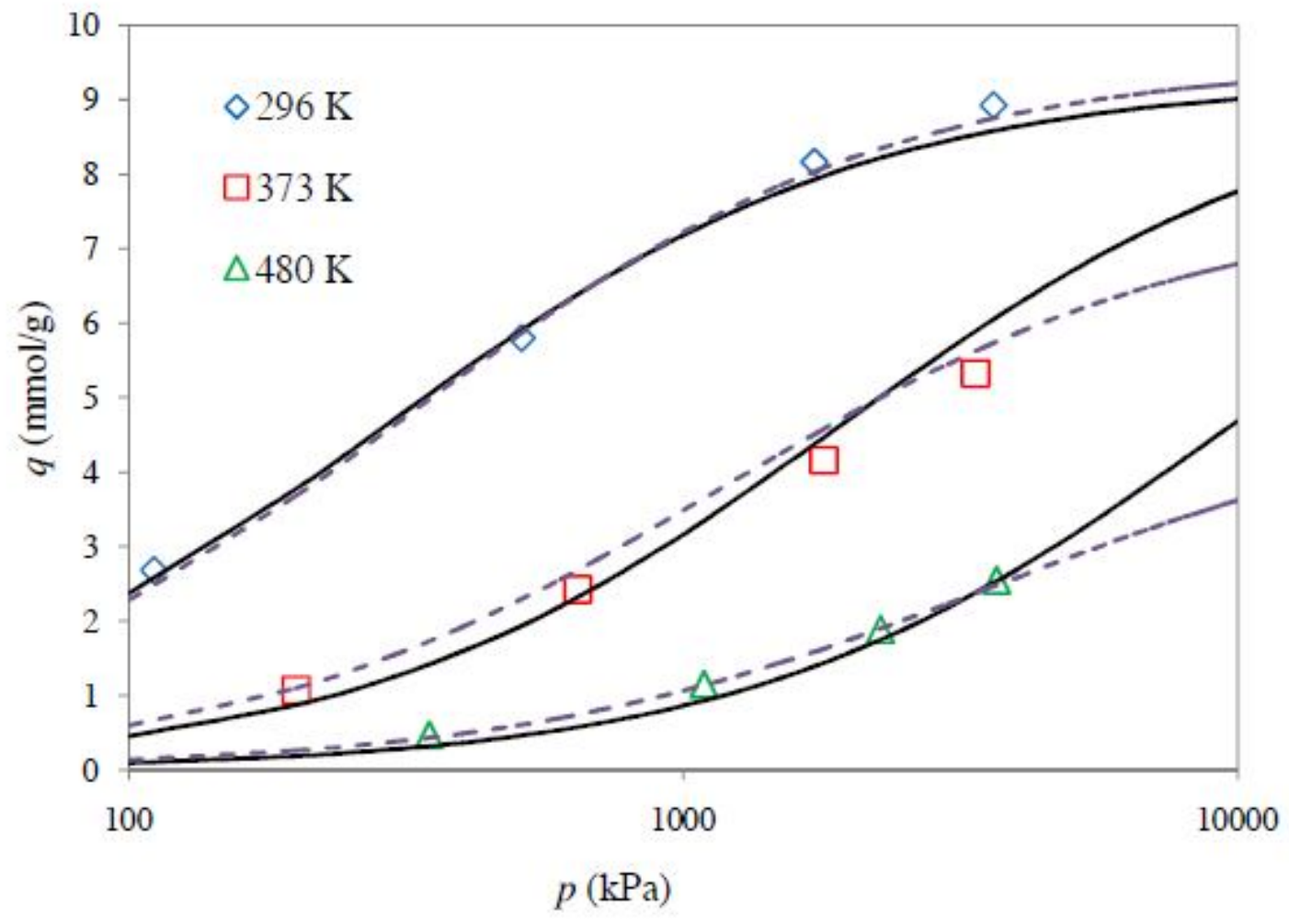

Fig. 10 


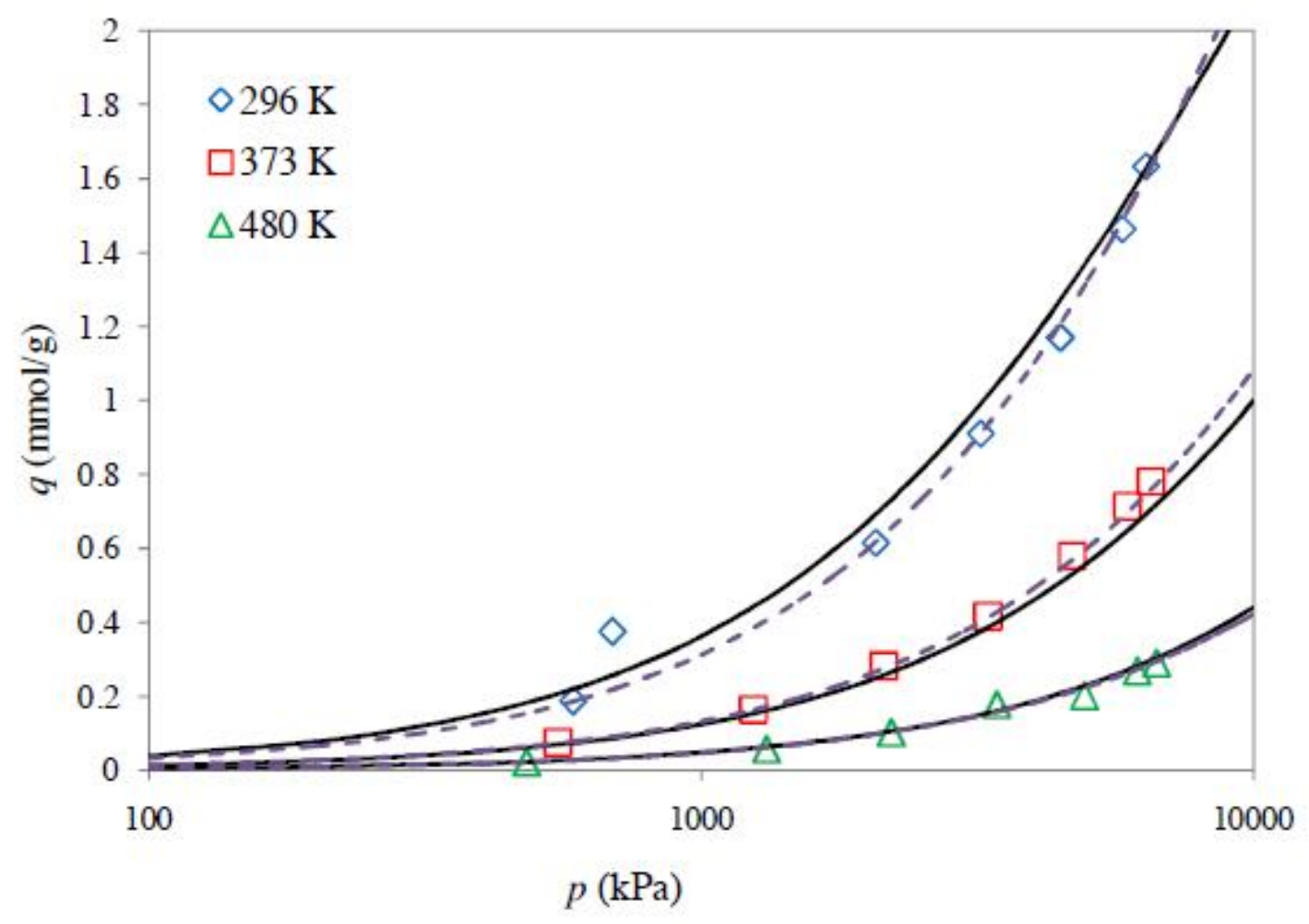

Fig. 11 


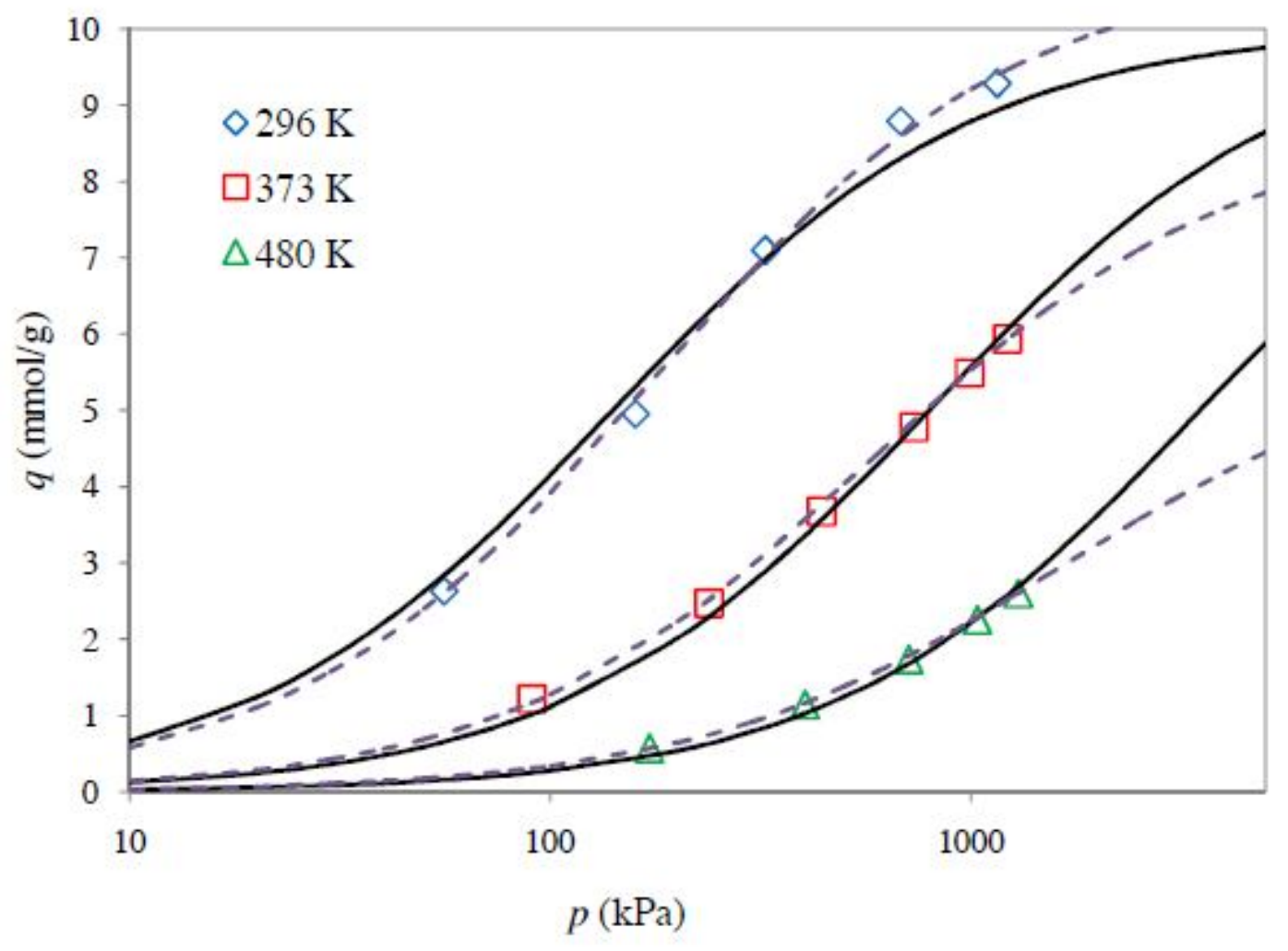

Fig. 12 


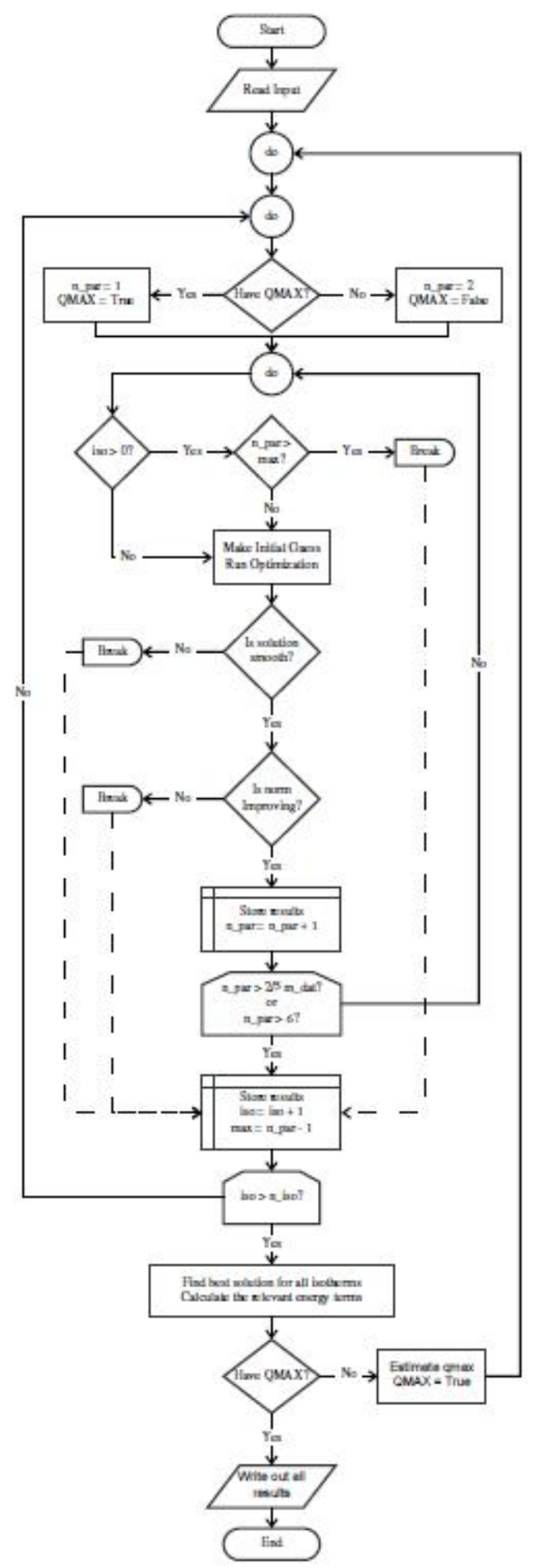

Fig. A1 